\title{
Tight contact structures and genus one fibered knots
}

\author{
JOHN A BALDWIN
}

\begin{abstract}
We study contact structures compatible with genus one open book decompositions with one boundary component. Any monodromy for such an open book can be written as a product of Dehn twists around dual nonseparating curves in the oncepunctured torus. Given such a product, we supply an algorithm to determine whether the corresponding contact structure is tight or overtwisted for all but a small family of reducible monodromies. We rely on Ozsváth-Szabó Heegaard Floer homology in our construction and, in particular, we completely identify the $L$-spaces with genus one, one boundary component, pseudo-Anosov open book decompositions. Lastly, we reveal a new infinite family of hyperbolic three-manifolds with no co-orientable taut foliations, extending the family discovered by Roberts, Shareshian, and Stein in [24].
\end{abstract}

57M27, 57R17, 57R58; 57R30

\section{Introduction}

Let $\operatorname{Aut}(\Sigma, \partial \Sigma)$ denote the set of isotopy classes of orientation preserving diffeomorphisms of $\Sigma$ which restrict the identity on $\partial \Sigma$ (where isotopies also restrict to the identity on $\partial \Sigma$ ). When $\Sigma$ is a once-punctured torus, $\operatorname{Aut}(\Sigma, \partial \Sigma)$ is generated by right-handed Dehn twists about dual nonseparating curves, $x$ and $y$. In an abuse of notation we denote by $\gamma$ the right-handed Dehn twist around the curve $\gamma \subset \Sigma$. The left-handed Dehn twist around $\gamma$ is then denoted by $\gamma^{-1}$. When it is unclear whether we are talking about a curve or a Dehn twist, we will use the notation $D_{\gamma}$ for the right-handed twist around $\gamma$. Given an open book decomposition $(\Sigma, \phi)$, we can express $\phi$ as a product of Dehn twists, $x^{a_{1}} y^{b_{1}} x^{a_{2}} y^{b_{2}} \cdots x^{a_{n}} y^{b_{n}}$, with $a_{i}, b_{j} \in \mathbb{Z}$, where composition is on the left.

There is an equivalence relation on open books given by positive stabilization/destabilization, and Giroux [6], extending results of Thurston and Winkelnkemper [27], showed that equivalence classes of open books are in one-to-one correspondence with isotopy classes of contact structures. Therefore, given a contact structure compatible with an open book, it is natural to ask whether we can infer properties of the contact structure simply by examining its monodromy. For instance, Giroux proved that a contact structure is Stein fillable, and hence tight, if it has a compatible open book whose 
monodromy is the product of right-handed Dehn twists [6]. The converse is also true. In general, however, there are tight contact structures which are not Stein fillable (or even symplectically fillable, as shown by Etnyre and Honda [4]).

Along these lines, we give an algorithm which explicitly determines when a contact structure compatible with a genus one open book with one boundary component is tight and when it is overtwisted. Our algorithm works for all but a small family of reducible monodromies. The input to the algorithm is a monodromy, written as a word in the Dehn twists $x$ and $y$. First, we state the main result when $\phi$ is pseudo-Anosov. ${ }^{1}$ Recall that pseudo-Anosov is equivalent, in the case of the once-punctured torus, to the condition that $\left|\operatorname{trace}\left(\phi_{\#}\right)\right|>2$, where $\phi_{\#}: H_{1}(\Sigma, \mathbb{Z}) \longrightarrow H_{1}(\Sigma, \mathbb{Z})$ is the induced map on homology. Pseudo-Anosov monodromies are especially interesting because the associated mapping torus $M_{\phi}$ is hyperbolic if $\phi$ is pseudo-Anosov, by a result of Thurston [25]. We will return to this fact in Section 7. From this point forward, $\Sigma$ will denote a genus one surface with one boundary component. The following is merely a preliminary theorem which makes subsequent calculation much easier.

Theorem 1.1 Let $\delta$ be a curve parallel to the boundary, and let $\phi$ be pseudo-Anosov. Then the open book $(\Sigma, \phi)$ is equal to an open book whose monodromy is of the following form:

(I) $\delta^{k} \cdot x^{a_{1}} y^{-b_{1}} \cdots x^{a_{n}} y^{-b_{n}}$, if $\operatorname{trace}(\phi)>2$

(II) $\delta^{k} \cdot x y^{2} x y^{2} \cdot x^{a_{1}} y^{-b_{1}} \cdots x^{a_{n}} y^{-b_{n}}$, if $\operatorname{trace}(\phi)<-2$

Here $k \in \mathbb{Z}, a_{i}, b_{j} \in \mathbb{Z}^{\geq 0}$, and $a_{i} \neq 0 \neq b_{j}$ for some $i, j$.

The pseudo-Anosov version of our main theorem is:

Theorem 1.2 Let $\phi$ be a pseudo-Anosov, boundary-fixing automorphism of the oncepunctured torus. If $\phi$ is of type I then the contact structure compatible with $(\Sigma, \phi)$ is tight if and only if $k \geq 1$. Likewise, if $\phi$ is of type II then the contact structure compatible with $(\Sigma, \phi)$ is tight if and only if $k \geq 0$.

We generalize both of these theorems in later sections to account for all monodromies.

To place this result in its proper context, it is necessary to discuss the recent work of Honda, Kazez, and Matić. In late 2005, they found a general criterion for the tightness of an open book, introducing the notion of right-veering diffeomorphisms [9]. Their result

\footnotetext{
${ }^{1}$ Throughout, when we say that $\phi$ is pseudo-Anosov we really mean that $\phi$ is isotopic to a pseudoAnosov diffeomorphism via a free isotopy - that is, one which does not necessarily restrict to the identity on $\partial \Sigma$.
} 
is an improvement over Goodman's sobering arc criterion for overtwistedness [7]. In particular, they prove that a contact structure $\xi$ is tight if and only if all of its compatible open books $(\Sigma, \phi)$ have right-veering $\phi$.

In general, however, it is very difficult to prove statements about all open books compatible with a given contact structure. Our paper succeeds in characterizing tightness for contact structures in terms of a single compatible open book, when the open book has genus equal to one and one boundary component, for almost all monodromies. Honda, Kazez, and Matić have since succeeded in characterizing tightness in terms of a single compatible open book (of genus one with one boundary component), for all monodromies. Their results are phrased in terms of the fractional Dehn twist coefficient of $\phi$, and can be found in $[8 ; 9 ; 10]$. Our approach is entirely different, and it is unique in the fact that we provide an explicit algorithm for detecting tightness when given a monodromy written as a product of Dehn twists of the sort described above. Moreover, our method leads very naturally to the discovery of a new family of hyperbolic threemanifolds with no taut foliations, as well as to a complete identification of Heegaard Floer homology $L$-spaces among those three-manifolds which contain a genus one fibered knot with pseudo-Anosov monodromy. In particular we are able to verify, for three-manifolds which contain a genus one fibered knot, a conjecture of Ozsváth and Szabó about $L$-spaces whose first homology groups have order one. In addition, an understanding of the Heegaard Floer homology groups of some of these $L$-spaces facilitates the computation of the Hopf invariants of their related contact structures.

Another interesting and related project is to identify those monodromies that give tight contact structures (denote this set by Tight $(\Sigma, \partial \Sigma)$ ), but which cannot be expressed as the product of right-handed Dehn twists along curves on the once-punctured torus (denote this set by $\operatorname{Dehn}^{+}(\Sigma, \partial \Sigma)$ ). This is the central topic of [10], and it is a step towards characterizing monodromies which produce tight, but not Stein fillable contact structures. An advantage of our explicit approach is that it allows us to easily identify a large family of monodromies in $\operatorname{Tight}(\Sigma, \partial \Sigma)-\operatorname{Dehn}^{+}(\Sigma, \partial \Sigma)$. The reader should compare these monodromies with those found in [10].

\subsection{Organization}

The organization of this paper is as follows: In Section 2, we prove a generalization of Theorem 1.1 by somewhat tedious manipulations in $\operatorname{Aut}(\Sigma, \partial \Sigma)$. In Section 3, we calculate the Ozsváth-Szabó contact invariants for the type II monodromies of Theorem 1.1 and we prove half of Theorem 1.2. In Section 4, we complete the proof of Theorem 1.2 using Goodman's criterion for overtwistedness. In addition, we generalize Theorem 1.2, giving a characterization of tightness for all genus one, one boundary 
component open books, with a small family of exceptions. Section 5 is a discussion of the Hopf invariants and $\operatorname{spin}^{c}$ structures associated to these contact structures. In Section 6, we analyze Tight $(\Sigma, \partial \Sigma)-\operatorname{Dehn}^{+}(\Sigma, \partial \Sigma)$. Finally, in Section 7 we classify $L$-spaces which have genus one, one boundary component open book decompositions. This involves a comparison with some of Roberts' results on taut foliations [22; 23]. Moreover, we identify an infinite family of hyperbolic $L$-spaces obtained by surgery on the bindings of these open books. Section 8 is an Appendix containing the proof of Lemma 3.6.

Acknowledgements I wish to thank Shaffiq Welji, Elisenda Grigsby, Jiajun Wang, Matt Hedden, and Joan Licata for helping me understand Heegaard Floer homology. I am also grateful to John Etnyre and Rachel Roberts for enlightening e-mail discussions. Most of all, I am indebted to my advisor, Peter Ozsváth, who suggested that I study the Heegaard Floer homology of these open books. Rarely have I left his office without a new idea.

\section{Proof of Theorem 1.1}

\subsection{Useful notation and the mapping class group}

This section is devoted to proving a generalization of Theorem 1.1. The details are somewhat tedious, so feel free to skip ahead to the statement of Theorem 2.6. As mentioned in the Introduction, the mapping class group, $\operatorname{Aut}(\Sigma, \partial \Sigma)$, of the oncepunctured torus is generated by right-handed Dehn twists about dual nonseparating curves, $x, y \subset \Sigma$. We orient $x$ and $y$ so that $i(x, y)=+1$, where $i$ is the intersection form on $H_{1}(\Sigma, \mathbb{Z})$. Also, we let $\delta$ denote a curve in $\Sigma$ parallel to the boundary. Given an open book $(\Sigma, \phi)$, where $\phi$ is a word in $\operatorname{Aut}(\Sigma, \partial \Sigma)$, it is useful to know how we can change $\phi$ and preserve the open book. We will use the following relations from $\operatorname{Aut}(\Sigma, \partial \Sigma)$ :

- $x y x=y x y$

- $\left(x y^{2} x y^{2}\right)^{2}=(x y)^{6}=\delta$

- If $\gamma$ and $\tau$ are disjoint curves in $\Sigma$, then $\gamma \tau=\tau \gamma$.

From the first of these relations, it follows that the word $h=x y^{2} x y^{2}=(x y)^{3}$ commutes with every other word in $\operatorname{Aut}(\Sigma, \partial \Sigma) .^{2}$ To verify this, we only need to show that $h$

\footnotetext{
${ }^{2}$ The letter $h$ is used to denote this word because $h$ is freely isotopic to the hyperelliptic involution of $\Sigma-$ it represents half a twist around the boundary.
}

Algebraic 83 Geometric Topology, Volume 7 (2007) 
commutes with both $x$ and $y$. We show that $h$ commutes with $x$, and the proof that $h$ commutes with $y$ is virtually identical:

$$
h \cdot x=x y y x y y x=x y x y x y x=x x y x y x y=x x y y x y y=x \cdot h .
$$

The following notational convention will be useful. Let $M\left(k ; b_{1}, \ldots, b_{n}\right)$ denote the open book $\left(\Sigma, \delta^{k} \cdot x y^{b_{1}} x y^{b_{2}} \cdots x y^{b_{n}}\right)$ for any collection of $b_{i} \in \mathbb{Z}$. Then Theorem 1.1 has the following reformulation:

Theorem 1.1 Let $\phi$ be pseudo-Anosov. Then the open book $(\Sigma, \phi)$ is equal to an open book whose monodromy is of the following form:

(I) $M\left(k ;-b_{1}, \ldots,-b_{n}\right)$, if $\operatorname{trace}(\phi)>2$

(II) $M\left(k ; 2,2,-b_{1}, \ldots,-b_{n}\right)$, if $\operatorname{trace}(\phi)<-2$

Here $k \in \mathbb{Z}, b_{j} \in \mathbb{Z}^{\geq 0}$, and $b_{i} \neq 0$ for some $i$.

Below is a list of moves which change the word $\phi$, but preserve the open book $(\Sigma, \phi)$. Each is obtained from a combination of the relations mentioned above, together with the observation that $\left(\Sigma, w_{1} \cdot w_{2}\right)$ is the same open book as $\left(\Sigma, w_{2} \cdot w_{1}\right)$, where $w_{1}, w_{2}$ are words in $\operatorname{Aut}(\Sigma, \partial \Sigma)$ - in other words, conjugation does not change the open book. This is not a manifestation of relations in $\operatorname{Aut}(\Sigma, \partial \Sigma)$, but rather it is due to the fact that the open book $(\Sigma, \phi)$ is constructed from the mapping torus, $M_{\phi}$.

Lemma 2.1 The following moves preserve the open books:

(1) $M\left(k ; b_{1}, \ldots, b_{n}\right)=M\left(k ; b_{2}, \ldots, b_{n}, b_{1}\right)$

(2) $M(k ; b_{1}, \ldots, \overbrace{b_{i}, 1, b_{i+1}}, \ldots, b_{n})=M(k ; b_{1}, \ldots, \overbrace{b_{i}+1, b_{i+1}+1}, \ldots, b_{n})$

(3) $M(k ; b_{1}, \ldots, \overbrace{b_{i}, 2,2,2,2, b_{i+1}}, \ldots, b_{n})$

$$
=M(k ; b_{1}, \ldots, \overbrace{b_{i}, 1,1,1,1,1,1, b_{i+1}}, \ldots, b_{n})=M\left(k+1 ; b_{1}, \ldots, b_{n}\right)
$$

(4) $M(k ; b_{1}, \ldots, \overbrace{b_{i}, 2, b_{i+1}}, \ldots, b_{n})=M(k ; b_{1}, \ldots, \overbrace{b_{i} \pm m, 2, b_{i+1} \mp m}, \ldots, b_{n})$

(5) $M(k ; b_{1}, \ldots, \overbrace{b_{i}, 2,2, b_{i+1}}, \ldots, b_{n})=M(k ; 2,2, b_{1}, \ldots, \overbrace{b_{i}, b_{i+1}}, \ldots, b_{n})$

(6) $M(k ; b_{1}, \ldots, \overbrace{b_{i}, 3, b_{i+1}}, \ldots, b_{n})=M(k ; 2,2, b_{1}, \ldots, \overbrace{b_{i}-1, b_{i+1}-1}, \ldots, b_{n})$

Proof of Lemma 2.1 (1), (2), and (3) follow trivially from the relations that we have discussed above. (4) follows because

$$
\begin{aligned}
M\left(k ; b_{1}, \ldots, b_{i}, 2, b_{i+1}, \ldots, b_{n}\right) & =M\left(k ; b_{1}, \ldots, b_{i}-1,1,1, b_{i+1}, \ldots, b_{n}\right) \\
& =M\left(k ; b_{1}, \ldots, b_{i}-1,2, b_{i+1}+1, \ldots, b_{n}\right)
\end{aligned}
$$


on one hand, using moves of type 2 . On the other hand,

$$
\begin{aligned}
M\left(k ; b_{1}, \ldots, b_{i}, 2, b_{i+1}, \ldots, b_{n}\right) & =M\left(k ; b_{1}, \ldots, b_{i}, 1,1, b_{i+1}-1, \ldots, b_{n}\right) \\
& =M\left(k ; b_{1}, \ldots, b_{i}+1,2, b_{i+1}-1, \ldots, b_{n}\right) .
\end{aligned}
$$

(5) follows from repeated applications of (4) or, if you prefer, from the fact that $h=x y^{2} x y^{2}$ commutes with $x$ and $y$, and thus with everything in $\operatorname{Aut}(\Sigma, \partial \Sigma)$. (6) follows from applications of (4) and (5):

$$
\begin{aligned}
M\left(k ; b_{1}, \ldots, b_{i}, 3, b_{i+1}, \ldots, b_{n}\right) & =M\left(k ; b_{1}, \ldots, b_{i}-1,1,2, b_{i+1}, \ldots, b_{n}\right) \\
& =M\left(k ; b_{1}, \ldots, b_{i}-1,2,2, b_{i+1}-1, \ldots, b_{n}\right) \\
& =M\left(k ; 2,2, b_{1}, \ldots, b_{i}-1, b_{i+1}-1, \ldots, b_{n}\right) .
\end{aligned}
$$

Lemma 2.2 Every open book $(\Sigma, \phi)$ can be expressed in the form $M\left(k ; b_{1}, \ldots, b_{2 n}\right)$ for $k, b_{i} \in \mathbb{Z}$.

Note that this is weaker than Theorem 1.1 which requires that the $b_{i} \leq 0$ and some $b_{j} \neq 0$.

\section{Proof of Lemma 2.2}

Claim 2.3 $x^{m}=\delta^{-1} \cdot x y x y x y x y x y^{m+1} x y=h^{-1} \cdot y x y^{m+1} x y$ for $m \in \mathbb{Z}$.

Certainly for $m=1$, we have:

$$
\begin{aligned}
x=\delta^{-1} \cdot x y x y x y x y x y x y x & =\delta^{-1} \cdot x y x y x y x y x y^{2} x y \\
& =\delta^{-1} \cdot h \cdot x y x y^{2} x y=h^{-1} y x y^{3} x y
\end{aligned}
$$

Now induct:

$$
\begin{aligned}
x^{m+1} & =x^{m} \cdot x \\
& =\delta^{-1} \cdot x y x y x y x y x y^{m+1} x y \cdot \delta^{-1} \cdot x y x y x y x y x y^{2} x y \\
& =\delta^{-2} \cdot x y x y x y x y x y^{m+1} x y x y x y x y x y x y^{2} x y \\
& =\delta^{-2} \cdot x y x y x y x y x y^{m+1} \cdot \delta \cdot y x y \\
& =\delta^{-1} \cdot x y x y x y x y x y^{m+2} x y \\
& =\delta^{-1} \cdot h \cdot x y x y^{m+1} x y \\
& =h^{-1} \cdot y x y^{m+3} x y
\end{aligned}
$$

Thus, for any $\phi=x^{a_{1}} y^{b_{1}} \cdots x^{a_{n}} y^{b_{n}},(\Sigma, \phi)$ is equal to the open book

$M\left(-n ; 1,1,1,1, a_{1}+1, b_{1}+1,1,1,1,1, a_{2}+1, b_{2}+1, \ldots, 1,1,1,1, a_{n}+1, b_{n}+1\right)$, 
completing the proof of Lemma 2.2. Note that this can be reduced with a combination of the moves 2 and 5 of Lemma 2.1 to $M\left(-n / 2 ; a_{1}+2, b_{1}+2, \ldots, a_{n}+2, b_{n}+2\right)$, for $n$ even, and $M\left(-n-1 / 2 ; 2,2, a_{1}+2, b_{1}+2, \ldots, a_{n}+2, b_{n}+2\right)$, for $n$ odd.

\subsection{Proof of Theorem 1.1}

Remark 2.4 We can use the comments immediately following Claim 2.3 to give another move among open books:

$$
\begin{aligned}
M\left(k ; b_{1}, \ldots, b_{2 n}\right) & =M\left(k-2 n ; 1,1,1,1,2, b_{1}+1, \ldots, 1,1,1,1,2, b_{2 n}+1\right) \\
& =M\left(k-n ; 3, b_{1}+2, \ldots, 3, b_{2 n}+2\right) .
\end{aligned}
$$

Therefore, any open book can be written as $M\left(k ; b_{1}, \ldots, b_{2 n}\right)$ by Lemma 2.2. Repeated applications of the above move show that $M\left(k ; b_{1}, \ldots, b_{2 n}\right)=M\left(d ; p_{1}, p_{2}, \ldots, p_{m}\right)$ for some $d$ and some collection of $p_{i} \geq 3$.

Lemma 2.5 $M\left(k ; b_{1}, \ldots, b_{n}\right)$ can be written as one of the following types of open books, via the word moves detailed above:

(1) $M\left(d ; p_{1}, \ldots, p_{m}\right)$, where the $p_{i} \geq 4$

(2) $M\left(d ; 2,2, p_{1}, \ldots, p_{m}\right)$, where the $p_{i} \geq 4$

(3) $M\left(d ; 2, p_{1}\right)$, where $p_{1} \geq 2$

(4) $M\left(d ; 2,2,2, p_{1}\right)$, where $p_{1} \geq 2$

(5) $M\left(d ; p_{1}\right)$, where $p_{1} \geq 1$

(6) $M\left(d ; 2,2, p_{1}\right)$, where $p_{1} \geq 1$

Proof of Lemma 2.5 Suppose that our open book is of the form $M\left(d ; p_{1}, \ldots, p_{m}\right)$ (or $M\left(d ; 2,2, p_{1}, \ldots, p_{m}\right)$ ) where the $p_{i} \geq 3$. Any open book can be written in this form by Remark 2.4. We perform the following iteration:

Step 1 If all of the $p_{i} \geq 4$, then we can stop as we are in type 1 (or 2). If one of the $p_{i}=3$, and $m \geq 3$, then go to Step 2. Otherwise, stop.

Step 2 Apply the word move in Lemma 2.1(6) to obtain $M\left(d ; 2,2, p_{1}, \ldots, p_{i-1}-\right.$ $\left.1, p_{i+1}-1, \ldots, p_{m}\right)$ (or $\left.M\left(d+1 ; p_{1}, \ldots, p_{i-1}-1, p_{i+1}-1, \ldots, p_{m}\right)\right)$. If neither $p_{i-1}-1$ nor $p_{i+1}-1$ is equal to 2 then return to Step 1 . If both $p_{i-1}-1, p_{i+1}-1=2$, and $m \geq 4$ then go to Step 3 . If exactly one of $p_{i-1}-1, p_{i+1}-1$ is 2 , say $p_{i-1}-1=2$, and $m \geq 4$, then go to Step 4. Otherwise, stop.

Step 3 Apply the word moves in Lemma 2.1 to obtain the open book $M(d+$ $\left.1 ; p_{1}, \ldots, p_{i-2}, p_{i+2}, \ldots, p_{m}\right)$ (or $M\left(d+1 ; 2,2, p_{1}, \ldots, p_{i-2}, p_{i+2}, \ldots, p_{m}\right)$ ). Return to Step 1. 
Step 4 Our open book is of the form $M\left(d ; 2,2, p_{1}, \ldots, 2, p_{i+1}-1, \ldots, p_{m}\right)$ (or $M(d+$ $\left.\left.1 ; p_{1}, \ldots, 2, p_{i+1}-1, \ldots, p_{m}\right)\right)=M\left(d ; 2,2, p_{1}, \ldots, 2,2, p_{i+1}-1+p_{i-2}-2, \ldots, p_{m}\right)$ $=M\left(d+1 ; p_{1}, \ldots, p_{i+1}-1+p_{i-2}-2, \ldots, p_{m}\right)\left(\right.$ or $M\left(d+1 ; 2,2, p_{1}, \ldots, p_{i+1}-1+\right.$ $\left.\left.p_{i-2}-2, \ldots, p_{m}\right)\right)$. Return to Step 1 .

All that remains of the proof is to show that this iteration stops exactly when we are in one of the types of Lemma 2.5. It stops at Step 1 if we ever reach a point in which our open book is $M\left(d ; p_{1}, \ldots, p_{m}\right)$ (or $\left.M\left(d ; 2,2, p_{1}, \ldots, p_{m}\right)\right)$ where the $p_{i} \geq 4$. Here, we are in type 1 (or 2). It stops at Step 1 if one of the $p_{i}=3$, and $m=2$. In this case, the open book looks like $M\left(d ; 3, p_{2}\right)\left(\right.$ or $\left.M\left(d ; 2,2,3, p_{2}\right)\right)=$ $M\left(d ; 2,1, p_{2}-1\right)=M\left(d ; p_{2}-1,2,1\right)=M\left(d ; p_{2}-2,2,2\right)=M\left(d ; 2,2, p_{2}-2\right)$ (or $M\left(d+1 ; p_{2}-2\right)$ ), and we are in type 6 (or 5). It stops at Step 1 if one of the $p_{i}=3$, and $m=1$, in which case we are in type 5 or 6 .

The iteration stops at Step 2 if $p_{i}=3$, both $p_{i-1}-1, p_{i+1}-1=2$, and $m=3$. In this case, the open book looks like $M(d ; 2,2,2,2)($ or $M(d+1 ; 2,2))$, and we are in type 3 (or 4 ). Finally, it stops at Step 2 if one of the $p_{i}=3$, exactly one of $p_{i-1}-1, p_{i+1}-1$ is equal to 2 (say $p_{i-1}-1=2$ ), and $m=3$. In this case, the open book looks like $M\left(d ; 2,2,2, p_{i+1}-1\right)$ (or $\left.M\left(d+1 ; 2, p_{i+1}-1\right)\right)$, and we are in type 3 (or 4 ).

The following theorem generalizes Theorem 1.1.

Theorem 2.6 Any open book can be written as $(\Sigma, \phi)$, where $\phi$ is one of the following types:

(A) $\delta^{d} \cdot x^{a_{1}} y^{-1} \cdots x^{a_{n}} y^{-1}$, where the $a_{i} \geq 0$, some $a_{j} \neq 0$

(B) $\delta^{d} \cdot x y^{2} x y^{2} \cdot x^{a_{1}} y^{-1} \cdots x^{a_{n}} y^{-1}$, where the $a_{i} \geq 0$, some $a_{j} \neq 0$

(C) $\delta^{d} \cdot y^{m}$, for $m \in \mathbb{Z}$

(D) $\delta^{d} \cdot x y^{2} x y^{2} \cdot y^{m}$, for $m \in \mathbb{Z}$

(E) $\delta^{d} \cdot x^{m} y^{-1}$, where $m \in\{-1,-2,-3\}$

(F) $\delta^{d} \cdot x y^{2} x y^{2} \cdot x^{m} y^{-1}$, where $m \in\{-1,-2,-3\}$

Only types A and B are pseudo-Anosov.

Proof of Theorem 2.6 We simply need to show that monodromies of the open books in types 1-6 of Lemma 2.5 can be expressed as monodromies of types A-F. Using repeated applications of Claim 2.3, for $m$ even, we can write

$$
x y^{p_{1}} \cdots x y^{p_{m}}=\delta^{\frac{m}{2}} \cdot y^{-1} x^{p_{1}-3} y^{-1} x^{p_{2}-4} \cdots y^{-1} x^{p_{m-1}-4} y^{-1} x^{p_{m}-3} y^{-1} x^{-1} .
$$


To see this, simply substitute $x^{p_{i}-s}=h^{-1} y x y^{p_{i}+2-s} x y$ for each $i$ into the expression on the right and simplify, using the fact that $h$ commutes with everything. Likewise, for $m$ odd, we can write

$x y^{p_{1}} \cdots x y^{p_{m}}=\delta^{\frac{m+1}{2}} \cdot y^{-1} x^{p_{1}-3} y^{-1} x^{p_{2}-4} \cdots y^{-1} x^{p_{m-1}-4} y^{-1} x^{p_{m}-3} \cdot\left(y^{-1} x^{-1}\right)^{4}$.

Then we can substitute these identities into to the monodromies of types $1,2,5$, and 6, apply some of the relations discussed earlier, and see which of the types A-F we get. For monodromies of types 3 and 4, the reduction is easier. Observe that $x y^{2} x y^{p_{1}}=y^{0} x y^{2} x y^{p_{1}}=y^{2} x y^{2} x \cdot y^{p_{1}-2}=x y^{2} x y^{2} \cdot y^{p_{1}-2}$, and substitute. We give the results of these substitutions below.

(1) $M\left(d ; p_{1}, \ldots, p_{m}\right)=(\Sigma, \phi)$ where

$$
\phi= \begin{cases}\delta^{d+\frac{m}{2}} \cdot x^{p_{1}-4} y^{-1} \cdots x^{p_{m}-4} y^{-1} & \text { if } m \text { is even } \\ \delta^{d+\frac{m-1}{2}} \cdot x y^{2} x y^{2} \cdot x^{p_{1}-4} y^{-1} \cdots x^{p_{m}-4} y^{-1} & \text { if } m \text { is odd }\end{cases}
$$

(2) $M\left(d ; 2,2, p_{1}, \ldots, p_{m}\right)=(\Sigma, \phi)$ where

$$
\phi= \begin{cases}\delta^{d+\frac{m}{2}} \cdot x y^{2} x y^{2} \cdot x^{p_{1}-4} y^{-1} \cdots x^{p_{m}-4} y^{-1} & \text { if } m \text { is even } \\ \delta^{d+\frac{m+1}{2}} \cdot x^{p_{1}-4} y^{-1} \cdots x^{p_{m}-4} y^{-1} & \text { if } m \text { is odd }\end{cases}
$$

(3) $M\left(d ; 2, p_{1}\right)=(\Sigma, \phi)$ where $\phi=\delta^{d} \cdot x y^{2} x y^{2} \cdot y^{p_{1}-2}$

(4) $M\left(d ; 2,2,2, p_{1}\right)=(\Sigma, \phi)$ where $\phi=\delta^{d+1} \cdot y^{p_{1}-2}$

(5) $M\left(d ; p_{1}\right)=(\Sigma, \phi)$ where $\phi=\delta^{d} \cdot x y^{2} x y^{2} \cdot x^{p_{1}-4} y^{-1}$

(6) $M\left(d ; 2,2, p_{1}\right)=(\Sigma, \phi)$ where $\phi=\delta^{d+1} \cdot x^{p_{1}-4} y^{-1}$

Proof of Theorem 1.1 The proof of Theorem 1.1 is finished by the observation that only monodromies of types A and B are pseudo-Anosov. This is seen by computing $\operatorname{trace}\left(\phi_{\#}\right)$ for each of these types. If we let $([x],[y])$ be our basis for $H_{1}(\Sigma, \mathbb{Z})$, then

$$
\left(x^{m}\right)_{\#}=\left[\begin{array}{cc}
1 & m \\
0 & 1
\end{array}\right],\left(y^{m}\right)_{\#}=\left[\begin{array}{cc}
1 & 0 \\
-m & 1
\end{array}\right], \delta_{\#}=\left[\begin{array}{ll}
1 & 0 \\
0 & 1
\end{array}\right],\left(x y^{2} x y^{2}\right)_{\#}=\left[\begin{array}{cc}
-1 & 0 \\
0 & -1
\end{array}\right]
$$

The various word moves that we have illustrated certainly preserve trace $\left(\phi_{\#}\right)$. So, for an arbitrary $\phi \in \operatorname{Aut}(\Sigma, \partial \Sigma)$ it is clear that trace $\left(\phi_{\#}\right)>2$ if and only if $(\Sigma, \phi)$ is equal to an open book with type A monodromy. Likewise, $\operatorname{trace}\left(\phi_{\#}\right)<-2$ if and only if $(\Sigma, \phi)$ is equal to an open book with type B monodromy. Since these type A and B monodromies have the properties required by Theorem 1.1, the proof is complete.

Remark 2.7 Note that types $\mathrm{C}$ and D are reducible, while types $\mathrm{E}$ and $\mathrm{F}$ are periodic. 


\section{Computing the contact invariants}

\subsection{The contact invariant and surgery exact triangles}

To a contact three-manifold $(Y, \xi)$, Ozsváth and Szabó associate a class $c(\xi) \in$ $\widehat{H F}(-Y) /\{ \pm 1\}$, which is an invariant of the contact structure $\xi$ up to isotopy [15]. We will use $\mathbb{Z}_{2}$ coefficients throughout to avoid ambiguity in sign. This invariant encodes information related to the tightness of $\xi$. For instance, Ozsváth and Szabó prove that if $\xi$ is overtwisted, then $c(\xi)=0$. On the other hand, if $\xi$ is Stein fillable or strongly fillable, then $c(\xi) \neq 0[15 ; 17]$. The precise relationship between $c(\xi)$ and the tightness of $\xi$ is still unknown - there are tight contact structures with vanishing contact invariant, as shown by Ghiggini [5]. In fact, Lisca and Stipsicz conjecture that the contact invariant vanishes for contact structures with positive Giroux torsion [12]. Yet, we show that if $(Y, \xi)$ is compatible with a genus one, one boundary component open book $(\Sigma, \phi)$ (where $\phi$ is a monodromy of Theorem 2.6 and $\phi$ is not $x y^{2} x y^{2} \cdot y^{-k}$ for some $k>4$ ) then $c(\xi)=0$ if and only if $\xi$ is overtwisted. ${ }^{3}$ The contact invariant is defined in terms of a compatible open book decomposition, $c(\Sigma, \phi)$, and it satisfies the following property [15, Theorem 4.2]:

Theorem 3.1 If $(\Sigma, \phi)$ is an open book decomposition for $Y$, and $\gamma \subset Y-B$ is a curve supported in a page of the open book ( $B$ is the binding), which is not homotopic within the page to the boundary, then $\left(\Sigma, t_{\gamma}^{-1} \cdot \phi\right)$ induces an open book decomposition of $Y_{+1}(\gamma)$, and under the map

$$
F_{W}: \widehat{H F}(-Y) \longrightarrow \widehat{H F}\left(-Y_{+1}(\gamma)\right)
$$

obtained by the two-handle addition (and summing over all spin ${ }^{c}$ structures), we have that

$$
F_{W}(c(\Sigma, \phi))= \pm c\left(\Sigma, t_{\gamma}^{-1} \cdot \phi\right)
$$

In particular, this tells us that if $c(\Sigma, \phi) \neq 0$, then $c\left(\Sigma, t_{\gamma} \cdot \phi\right) \neq 0$, where $\left(\Sigma, t_{\gamma} \cdot \phi\right)$ is the induced open book decomposition of $Y_{-1}(\gamma)$.

In this section, we compute the contact invariants $c(\Sigma, \phi)$, where $\phi$ is a monodromy of type II in Theorem 1.1. Borrowing the notation from Section 2, the following theorem holds.

Theorem 3.2 If $b_{j} \in \mathbb{Z}^{\geq 0}$ and some $b_{i} \neq 0$ then $c\left(M\left(0 ; 2,2,-b_{1}, \ldots,-b_{n}\right)\right) \neq 0$.

\footnotetext{
${ }^{3}$ Again, we should note that Honda, Kazez, and Matić have since shown this for all monodromies of Theorem 2.6.
} 
Recall that in $\operatorname{Aut}(\Sigma, \partial \Sigma)$, we have $\delta=(x y)^{6}=\left(x y^{2} x y^{2}\right)^{2}$. Then Theorem 3.2 and Theorem 3.1 imply that for $k \geq 1$,

$$
c\left(M\left(k ;-b_{1}, \ldots,-b_{n}\right)\right) \neq 0 .
$$

Hence, the contact structure compatible with $M\left(k ;-b_{1}, \ldots,-b_{n}\right)$ is tight for $k \geq 1$. In exactly the same way $M\left(k ; 2,2,-b_{1}, \ldots,-b_{n}\right)$ is tight for $k \geq 0$. This proves half of Theorem 1.2.

Before we give the proof of Theorem 3.2, we must examine the Heegaard Floer homology of the three-manifolds underlying these open books. In the language of Heegaard Floer homology, a rational homology 3 -sphere $Y$ is an $L$-space if $\widehat{H F}(Y, s) \cong \mathbb{Z}$ for every $\operatorname{spin}^{c}$-structure $s$ on $Y$. Ozsváth and Szabó show that $L$-spaces are closed under surgeries in the following sense [16, Proposition 2.1]:

Theorem 3.3 Suppose $K \subset Y$ is a knot with framing $\lambda$ and

$$
\left|H_{1}\left(Y_{\lambda}(K)\right)\right|=\left|H_{1}(Y)\right|+\left|H_{1}\left(Y_{\mu+\lambda}(K)\right)\right|
$$

where $\mu$ denotes the meridian for the knot, $Y_{\lambda}(K)$ is the 3-manifold obtained from $Y$ by performing surgery on $Y$ along $K$ with framing $\lambda$, and $|G|$ is the order of the group $G$ if this order is finite. Then, if $Y$ and $Y_{\mu+\lambda}(K)$ are both $L$-spaces, so is $Y_{\lambda}(K)$. Furthermore, the map

$$
F_{W}: \widehat{H F}(Y) \longrightarrow \widehat{H F}\left(Y_{\lambda}(K)\right)
$$

obtained by the two-handle addition (and summing over all spin ${ }^{c}$ structures) is injective.

The surgery diagrams for these genus one, one boundary component open books are easy to describe - see Figure 1 for an example. Let $\widehat{M}\left(k ; a_{1}, \ldots, a_{m}\right)$ denote the oriented three-manifold underlying the open book $M\left(k ; a_{1}, \ldots, a_{m}\right)$. Then the manifold $\widehat{M}\left(0 ; 2,2,-b_{1}, \ldots,-b_{n}\right)$ has the surgery diagram illustrated in Figure 2 (this diagram resembles Figure 1 after an isotopy of the surgery curves). The knot $K$ in Figure 2 lies in a page of the open book $M\left(0 ; 2,2,-b_{1}, \ldots,-b_{n}\right)$, and +1 -surgery on $K$ yields the manifold $\widehat{M}\left(0 ; 2,2,-b_{1}, \ldots,-b_{n}-1\right)$ with induced open book decomposition $M\left(0 ; 2,2,-b_{1}, \ldots,-b_{n}-1\right)$. Then, by Theorem 3.2, the map

$$
\begin{aligned}
F_{W}: \widehat{H F}\left(-\widehat{M}\left(0 ; 2,2,-b_{1}, \ldots,-b_{n}\right)\right) & \rightarrow \widehat{H F}\left(-\widehat{M}\left(0 ; 2,2,-b_{1}, \ldots,-b_{n}-1\right)\right) \\
\text { takes } \quad c\left(M\left(0 ; 2,2,-b_{1}, \ldots,-b_{n}\right)\right) & \mapsto c\left(M\left(0 ; 2,2,-b_{1}, \ldots,-b_{n}-1\right)\right) .
\end{aligned}
$$




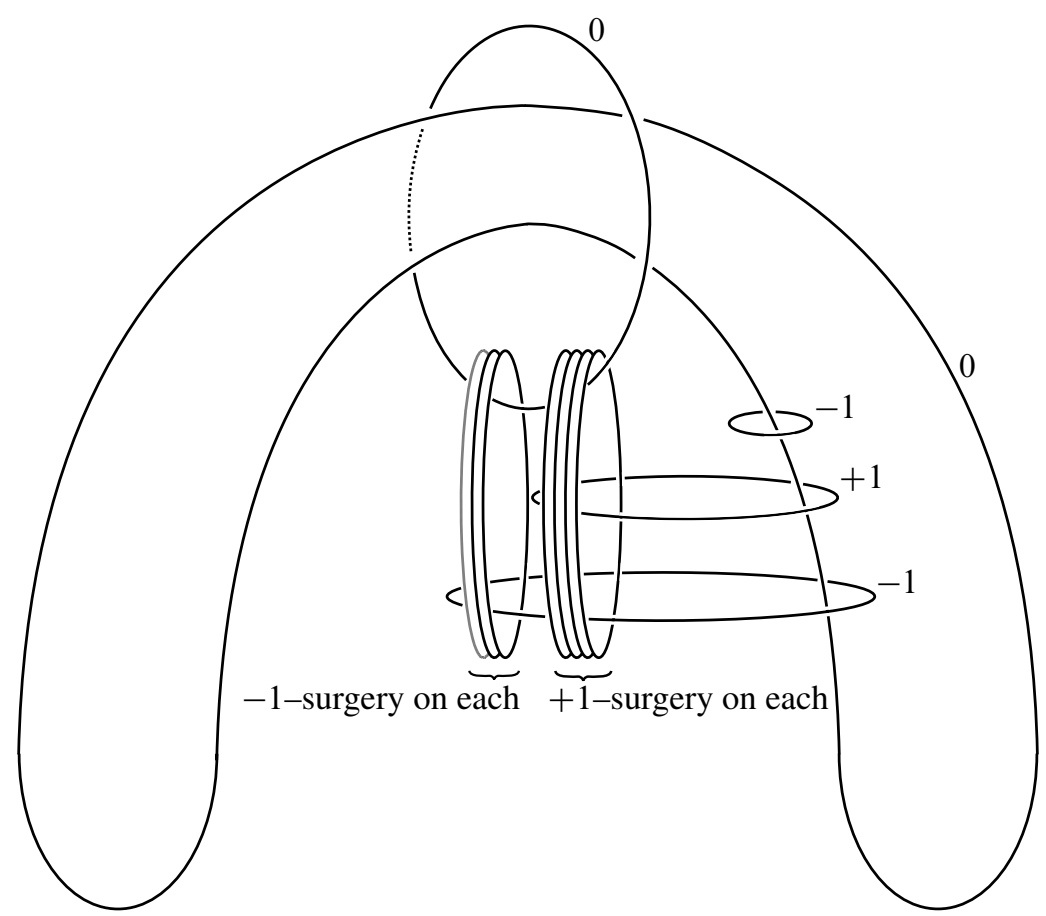

Figure 1: Surgery diagram for the open book with monodromy $\phi=x y^{-4} x^{-1} y^{3} x$

The manifolds $-\widehat{M}\left(0 ; 2,2,-b_{1}, \ldots,-b_{n}\right)$ and $-\widehat{M}\left(0 ; 2,2,-b_{1}, \ldots,-b_{n}-1\right)$ fit into a surgery exact triangle:

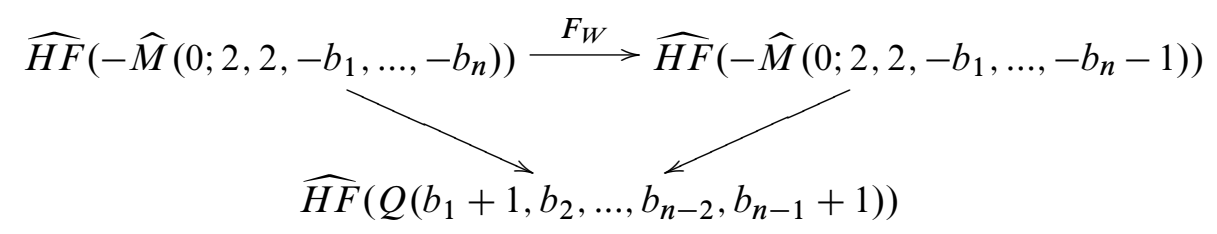

$Q\left(b_{1}, \ldots, b_{n}\right)$ is defined to be the three-manifold given by the surgery diagram in Figure 3. After a sequence of blowdowns and handleslides it can be shown that $Q\left(b_{1}+1, b_{2}, \ldots, b_{n-2}, b_{n-1}+1\right)$ is the manifold obtained by 0 -surgery on the knot $K$ in $-\widehat{M}\left(0 ; 2,2,-b_{1}, \ldots,-b_{n}-1\right)$.

Our calculation of the contact invariants depends on the following three lemmas:

Lemma 3.4 For $b_{j} \in \mathbb{Z}^{\geq 0}$ and some $b_{i} \neq 0, Q\left(b_{1}, \ldots, b_{n}\right)$ is an $L$-space.

Lemma 3.5 For $b_{j} \in \mathbb{Z}^{\geq 0}$ and some $b_{i} \neq 0,-\widehat{M}\left(0 ; 2,2,-b_{1}, \ldots,-b_{n}\right)$ is an $L-$ space. 


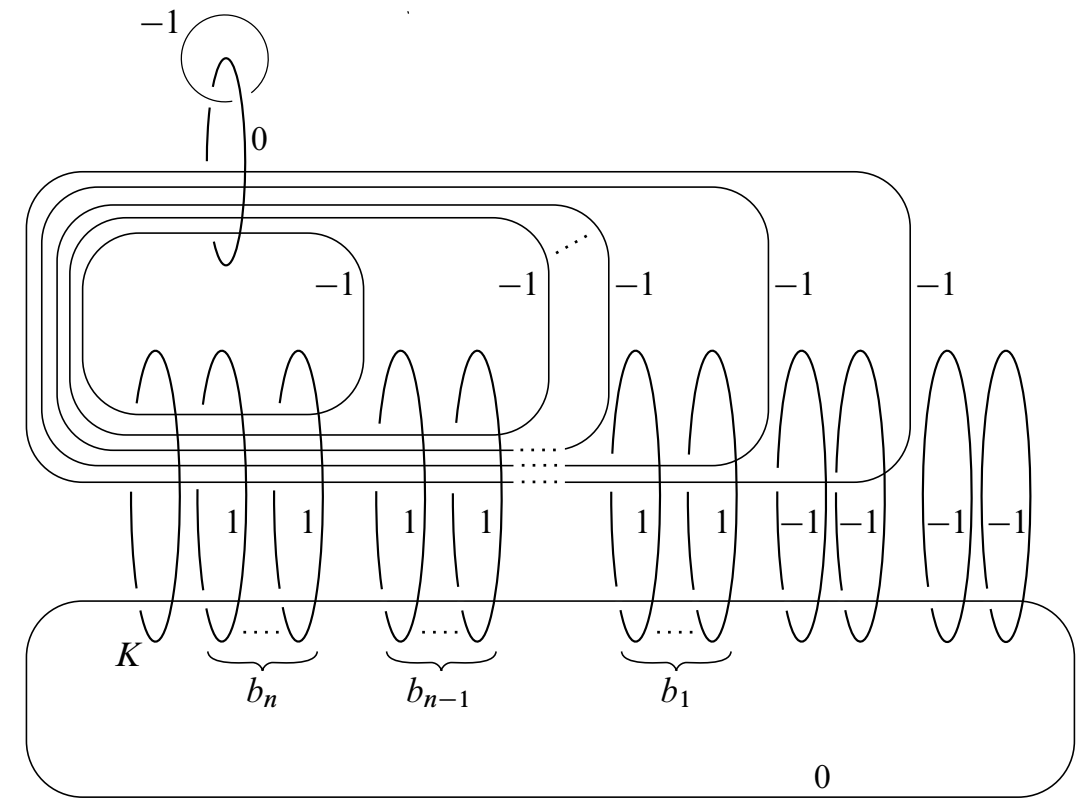

Figure 2: Surgery diagram for the $3-$ manifold $\widehat{M}\left(0 ; 2,2,-b_{1}, \ldots,-b_{n}\right)$

Lemma 3.6 For $b_{j} \in \mathbb{Z}^{\geq 0}$ and some $b_{i} \neq 0$,

$$
\begin{aligned}
\left|H_{1}\left(-\widehat{M}\left(0 ; 2,2,-b_{1}, \ldots,-b_{n}-1\right)\right)\right|= & \left|H_{1}\left(-\widehat{M}\left(0 ; 2,2,-b_{1}, \ldots,-b_{n}\right)\right)\right| \\
& +\left|H_{1}\left(Q\left(b_{1}+1, b_{2}, \ldots, b_{n-2}, b_{n-1}+1\right)\right)\right| .
\end{aligned}
$$

Theorem 3.2 follows immediately:

Proof of Theorem $3.2 M(0 ; 2,2,-1, \overbrace{0, \ldots, 0}^{m})=M(0 ; 2,2,0-1,0, \ldots, 0)=\cdots=$ $M(0 ; 2,2,0, \ldots, 0,-1)$ is the open book given by the monodromy $x y^{2} x y^{2} \cdot x y^{-1} x^{m}=$ $x x y x y x y \cdot y^{-1} x^{m}=x^{2} y x y x^{m+1}=x^{3} y x^{m+2}$. And $\left(\Sigma, x^{3} y x^{m+2}\right)=\left(\Sigma, y x^{m+5}\right)$. But then $M(0 ; 2,2,-1,0, \ldots, 0)$ is Stein fillable since it can be written as an open book whose monodromy consists solely of right-handed Dehn twists. Hence,

$$
c(M(0 ; 2,2,-1,0, \ldots, 0)) \neq 0 .
$$

Lemma 3.4-Lemma 3.6, together with Theorem 3.3 imply that $F_{W}$ is injective. Consequently,

$$
c\left(M\left(0 ; 2,2,-b_{1}, \ldots,-b_{n}\right)\right) \neq 0 \quad \Longrightarrow \quad c\left(M\left(0 ; 2,2,-b_{1}, \ldots,-b_{n}-1\right)\right) \neq 0 .
$$




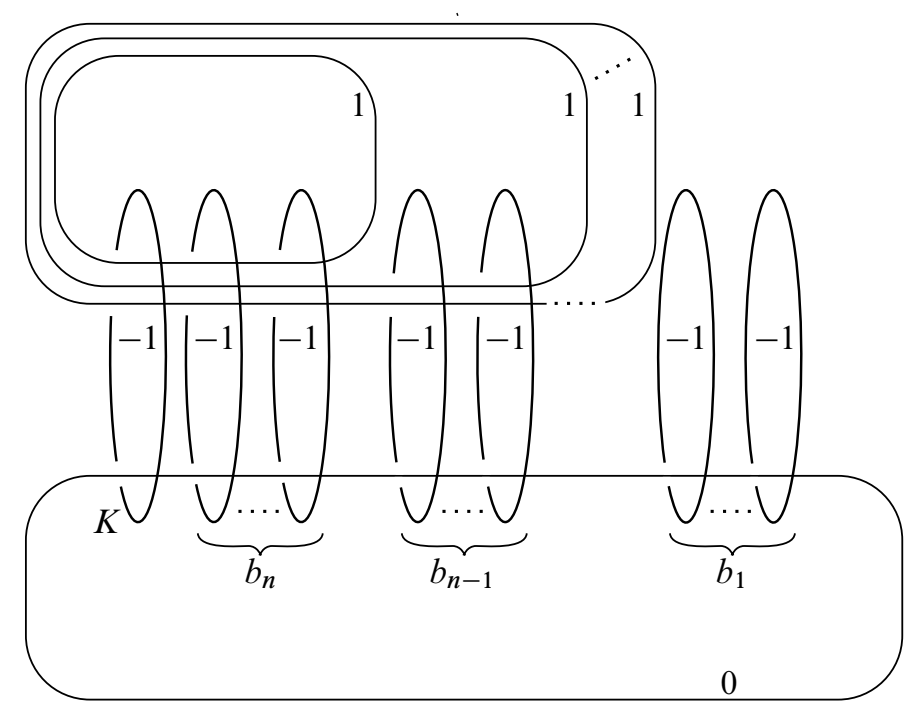

Figure 3: Surgery diagram for the 3-manifold $Q\left(b_{1}, \ldots, b_{n}\right)$

Since $c(M(0 ; 2,2,-1,0, \ldots, 0)) \neq 0$, we can induct (as in the later proofs of Lemma 3.4 and Lemma 3.5) to show that $c\left(M\left(0 ; 2,2,-b_{1}, \ldots,-b_{n}\right)\right) \neq 0$ for $b_{j} \in \mathbb{Z}^{\geq 0}$ and some $b_{i} \neq 0$, completing the proof of Theorem 3.2.

\subsection{Proof of Lemma 3.4-Lemma 3.6}

Lemma 3.6 follows from direct computation: we compare the determinants of the linking matrices of the surgery diagrams for the manifolds $-\widehat{M}\left(0 ; 2,2,-b_{1}, \ldots,-b_{n}\right)$, $-\widehat{M}\left(0 ; 2,2,-b_{1}, \ldots,-b_{n}-1\right)$, and $Q\left(b_{1}+1, b_{2}, \ldots, b_{n-2}, b_{n-1}+1\right)$. We save this proof for the Appendix.

Proof of Lemma 3.5 Proving Theorem 3.2, we noted that $M(0 ; 2,2,-1, \overbrace{0, \ldots, 0}^{m})$ is the open book $\left(\Sigma, y x^{m+5}\right)$ for some $m$. But then,

$$
\begin{aligned}
-\widehat{M}(0 ; 2,2,-1,0, \ldots, 0) & =-\widehat{M}(0 ; 2,2,0-1,0, \ldots, 0) \\
& =\cdots=-\widehat{M}(0 ; 2,2,0, \ldots, 0,-1) \\
& =-L(m+5,1)
\end{aligned}
$$

which is an $L$-space, as shown by Ozsváth and Szabó in [16]. Our proof proceeds by induction on the $b_{i}$. Suppose that $b_{i} \geq 1$ for some $i$ and either $b_{j}>1$ for some $j$, or $b_{k} \neq$ 0 for some $k \neq i$ (otherwise, $-\widehat{M}\left(0 ; 2,2,-b_{1}, \ldots,-b_{n}\right)=-\widehat{M}(0 ; 2,2,-1,0, \ldots, 0)$ ), 
then

$$
-\widehat{M}\left(0 ; 2,2,-b_{1}, \ldots,-b_{n}\right)=-\widehat{M}\left(0 ; 2,2,-b_{i+1}, \ldots,-b_{n},-b_{1}, \ldots,-b_{i}\right)
$$

and by induction, we know that $-\widehat{M}\left(0 ; 2,2,-b_{i+1}, \ldots,-b_{n},-b_{1}, \ldots,-\left(b_{i}-1\right)\right)$ is an $L$-space. Since $Q\left(b_{i+1}+1, \ldots, b_{n}, b_{1}, \ldots, b_{i-1}+1\right)$ is an $L$-space we can conclude, by Lemma 3.4 and Theorem 3.3, that

$$
-\widehat{M}\left(0 ; 2,2,-b_{1}, \ldots,-b_{n}\right)=-\widehat{M}\left(0 ; 2,2,-b_{i+1}, \ldots,-b_{n},-b_{1}, \ldots,-b_{i}\right)
$$

is an $L$-space.

Proof of Lemma 3.4 This proof is virtually identical in technique to that of Lemma 3.5. $Q\left(b_{1}, \ldots, b_{n-1}, b_{n}+1\right)$ is the manifold obtained by performing -1 -surgery on the knot $K$ in $Q\left(b_{1}, \ldots, b_{n}\right)$ (see Figure 3). Meanwhile, $Q\left(b_{1}, \ldots, b_{n-2}, b_{n-1}+1\right)$ is the manifold obtained by performing 0 -surgery on the knot $K$. The following claim is the analogue of Lemma 3.6 for these manifolds $Q$.

Claim 3.7 For $b_{j} \in \mathbb{Z}^{\geq 0}$ and some $b_{i} \neq 0$ then

$$
\left|H_{1}\left(Q\left(b_{1}, \ldots, b_{n-1}, b_{n}+1\right)\right)\right|=\left|H_{1}\left(Q\left(b_{1}, \ldots, b_{n}\right)\right)\right|+\left|H_{1}\left(Q\left(b_{1}, \ldots, b_{n-2}, b_{n-1}+1\right)\right)\right| \text {. }
$$

Again, this is proved directly by comparing the determinants of the linking matrices for these three manifolds. By Theorem 3.3, if $Q\left(b_{1}, \ldots, b_{n-2}, b_{n-1}+1\right)$ and $Q\left(b_{1}, \ldots, b_{n}\right)$ are $L$-spaces, then so is $Q\left(b_{1}, \ldots, b_{n-1}, b_{n}+1\right)$. To complete the proof of Lemma 3.4, we proceed by induction, as before.

Observe that $Q\left(0, a_{1}, \ldots, a_{m}\right)=Q\left(a_{1}, \ldots, a_{m}\right)=Q\left(a_{1}, \ldots, a_{m}, 0\right)$, and $Q(1)=S^{3}$, an $L$-space. Now, we induct on $n$ and on $b_{n}$ simultaneously. Consider $Q\left(b_{1}, \ldots, b_{n}\right)$. If $b_{n}=0\left(\right.$ or $\left.b_{1}=0\right)$, then $Q\left(b_{1}, \ldots, b_{n}\right)=Q\left(b_{1}, \ldots, b_{n-1}\right)$ (or $\left.Q\left(b_{2}, \ldots, b_{n}\right)\right)$ and we can conclude by our induction on $n$ that $Q\left(b_{1}, \ldots, b_{n}\right)$ is an $L$-space. If $b_{n} \neq 0$ and $b_{1} \neq 0$, then by our induction on $b_{n}, Q\left(b_{1}, \ldots, b_{n-1}, b_{n}-1\right)$ is an $L-$ space; and by induction on $n, Q\left(b_{1}, \ldots, b_{n-2}, b_{n-1}+1\right)$ is an $L$-space. Combining this with Claim 3.7 and Theorem 3.3, we can conclude that $Q\left(b_{1}, \ldots, b_{n}\right)$ is also an $L$-space. This completes the proof of Lemma 3.4, and, consequently, ties up the remaining loose end in the proof of Theorem 3.2 as well as half of Theorem 1.2.

Remark 3.8 There is actually a much shorter proof of Lemma 3.4: from their surgery diagrams, it is easy to see that these $Q$ manifolds have planar open book decompositions with monodromies which are composed of right-handed Dehn twists. In [21] Ozsváth and Szabó show that such three-manifolds are $L$-spaces. 
Remark 3.9 Using the inductive techniques above, we can prove that the manifolds $\widehat{M}\left(0 ;-b_{1}, \ldots,-b_{n}\right)$ are also $L-$ spaces for $b_{j} \in \mathbb{Z}^{\geq 0}$ and some $b_{i} \neq 0$. Again, this follows more easily from the observation that $\widehat{M}\left(0 ;-b_{1}, \ldots,-b_{n}\right)$ is the double cover of $S^{3}$ branched along the closed 3-braid specified by the word $\sigma_{1} \sigma_{2}^{-b_{1}} \ldots \sigma_{1} \sigma_{2}^{-b_{n}}$, where $\sigma_{1}$ and $\sigma_{2}$ are the standard generators of the braid group on three strands. This 3-braid is alternating, and it is shown by Ozsváth and Szabó in [19] that the double cover of $S^{3}$ branched along an alternating link is an $L$-space.

Remark 3.10 Since $Y$ is an $L$-space if and only if $-Y$ is an $L$-space, we can conclude that the three manifolds $\widehat{M}\left(0 ; 2,2,-b_{1}, \ldots,-b_{n}\right)$ are $L$-spaces, for $b_{j} \in \mathbb{Z}^{\geq 0}$ and some $b_{i} \neq 0$.

\section{Overtwistedness and sobering arcs}

In this section, we prove the second half of Theorem 1.2; that is, for $b_{j} \in \mathbb{Z}^{\geq 0}$ and some $b_{i} \neq 0, M\left(k ;-b_{1}, \ldots,-b_{n}\right)$ is overtwisted if $k<1$ and $M\left(k ; 2,2,-b_{1}, \ldots,-b_{n}\right)$ is overtwisted if $k<0$. These statements follow directly from Goodman's sobering arc criterion for overtwistedness [7]. First, a bit of background material.

Given two properly embedded oriented $\operatorname{arcs} \alpha, \beta$ with common boundary points in $\Sigma$, let $\beta^{\prime}$ be an arc transverse to $\alpha$ that minimizes intersections with $\alpha$ over boundaryfixing isotopies of $\beta$. Then let $i_{\text {alg }}(\alpha, \beta)$ denote the oriented intersection number of $\alpha$ with $\beta^{\prime}$, summed over points in the interiors of the arcs. Let $i_{\text {geom }}(\alpha, \beta)$ be the unsigned number of interior intersection points of $\alpha$ and $\beta^{\prime}$. And let $i_{\delta}(\alpha, \beta)$ be one-half the oriented sum of intersections at the boundaries of the $\operatorname{arcs} \alpha$ and $\beta^{\prime}$. In our case, suppose $\alpha$ is an arc in the page $\Sigma$ of an open book, $(\Sigma, \phi)$. Give the arc $\phi(\alpha)$ the orientation which is opposite the pushed-forward orientation of $\alpha$.

Definition 4.1 A properly embedded arc $\alpha \subset \Sigma$ is sobering for the monodromy $\phi$ if

$$
i_{\mathrm{alg}}(\alpha, \phi(\alpha))+i_{\text {geom }}(\alpha, \phi(\alpha))+i_{\delta}(\alpha, \phi(\alpha)) \leq 0
$$

and $\alpha$ is not isotopic to $\phi(\alpha)$.

See Figure 4 for an illustration of sobering arcs. Goodman showed the following:

Theorem 4.2 If there is a sobering arc $\alpha \subset \Sigma$ for the monodromy $\phi$, then the open book $(\Sigma, \phi)$ is overtwisted. 


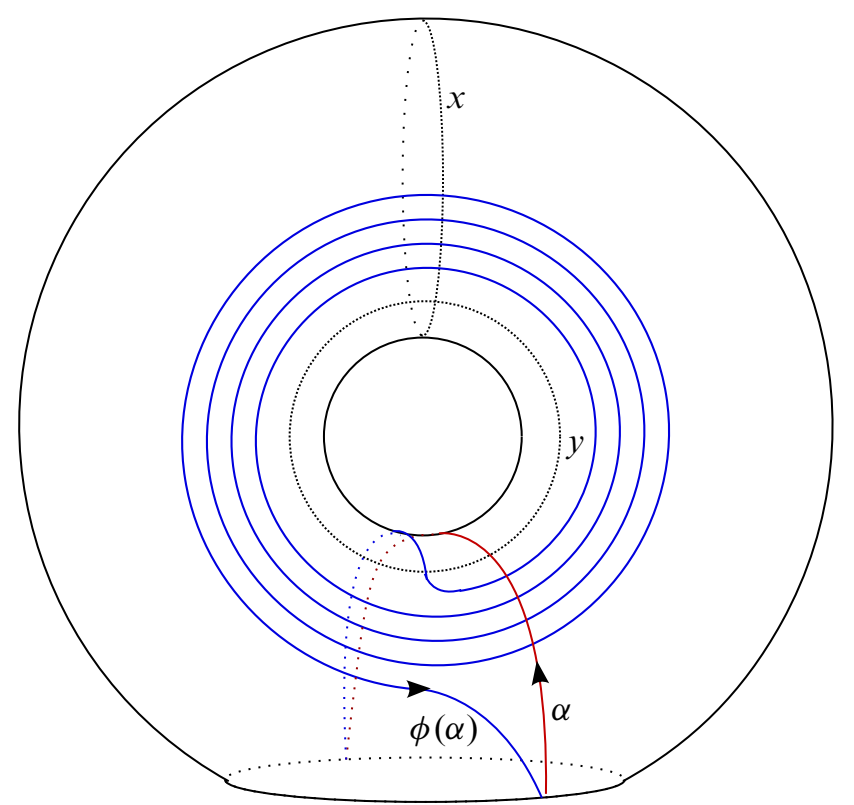

Figure 4: A sobering arc in $\alpha \subset \Sigma$ for the monodromy $\phi=y^{-4}$. Here $y$ is a longitude and $x$ a meridian.

Remark 4.3 Whenever a monodromy consists of at least one left-handed Dehn twist around $y$ and arbitrarily many right-handed Dehn twists around $x$, it is clear that an arc $\alpha$ which crosses the curve $y$ once and does not cross $x$ is sobering, as $i_{\text {alg }}(\alpha, \phi(\alpha))+i_{\text {geom }}(\alpha, \phi(\alpha))=0$, and $i_{\delta}(\alpha, \phi(\alpha))=-1$.

Remark 4.4 If $\alpha$ is an arc for the monodromy $\phi$ with

$$
i_{\text {alg }}(\alpha, \phi(\alpha))+i_{\text {geom }}(\alpha, \phi(\alpha))+i_{\delta}(\alpha, \phi(\alpha))=-1
$$

then $\alpha$ also satisfies

$$
i_{\text {alg }}\left(\alpha, h^{-1} \cdot \phi(\alpha)\right)+i_{\text {geom }}\left(\alpha, h^{-1} \cdot \phi(\alpha)\right)+i_{\delta}\left(\alpha, h^{-1} \cdot \phi(\alpha)\right)=-1
$$

where $h=x y^{2} x y^{2}$. This is because $h^{-1}$ is isotopic to a left-handed half Dehn twist around the boundary.

Proof of Theorem 1.2 The open book $M\left(0 ;-b_{1}, \ldots,-b_{n}\right)$ has monodromy which is the composition of left-handed Dehn twists around the curve $y$ with right-handed Dehn twists around the curve $x$, and is thus overtwisted by Remark 4.3. $M\left(k ;-b_{1}, \ldots,-b_{n}\right)$ has monodromy as in the case $k=0$, composed with $\delta^{k}=h^{2 k}$. Hence, by Remark 4.4, for $k<0, M\left(k ;-b_{1}, \ldots,-b_{n}\right)$ is overtwisted. 
Now consider the open book $M\left(k ; 2,2,-b_{1}, \ldots,-b_{n}\right)$. This open book has the same monodromy as the open book $M\left(0 ;-b_{1}, \ldots,-b_{n}\right)$, composed with $h^{2 k+1}$. So when $k<0, M\left(k ; 2,2,-b_{1}, \ldots,-b_{n}\right)$ is overtwisted by Remark 4.3 and Remark 4.4.

We generalized Theorem 1.1 in Theorem 2.6. Here we give the corresponding generalization of Theorem 1.2.

Theorem 4.5 A-F correspond to the monodromies of Theorem 2.6.
(A) Tight if and only if $d \geq 1$.
(B) Tight if and only if $d \geq 0$.
(C) Tight if and only if either $d>0$ or $d=0$ and $m \geq 0$.
(D) Tight if $d>0$ or $d=0$ and $m \geq-4$; overtwisted if $d<0.4$
(E) Tight if and only if $d \geq 1$.
(F) Tight if and only if $d \geq 0$.

Proof of Theorem 4.5 For types A and B, this statement is simply Theorem 1.2. The proof for types C-F is a combination of Goodman's sobering arc criterion (Theorem 4.2), Remark 4.3 and Remark 4.3, and Theorem 3.2, Theorem 3.1, and Theorem 1.2.

Type C If $d>0$, then $\delta^{d} \cdot y^{m}=\delta^{d-1} \cdot y^{2} x y^{2} \cdot x y^{2} x y^{2} \cdot x y^{m}$. Since we have $c\left(\Sigma, x y^{2} x y^{2} \cdot x y^{m}\right) \neq 0$ for any $m$ (by Theorem 3.2), we conclude by Theorem 3.1 that $c\left(\Sigma, \delta^{d} \cdot y^{m}\right) \neq 0$, hence this open book is tight.

If $d<0$, then an arc $\alpha$ which crosses $x$ once and does not intersect $y$ is sobering for the monodromy $\delta^{d} \cdot y^{m}$. Hence, the corresponding open book is overtwisted by Theorem 4.2.

If $d=0$, then $c\left(\Sigma, y^{m}\right) \neq 0$ if $m>0$ since the contact structure is Stein fillable. For $m=0$, we have the empty monodromy. Stabilizing once, we find that this is Stein fillable, hence tight. When $m<0, c\left(\Sigma, y^{m}\right)$ is overtwisted by Remark 4.3.

Type D If $d>0$, then $\delta^{d} \cdot x y^{2} x y^{2} \cdot y^{m}=\delta^{d-1} \cdot x y^{2} x y^{2} \cdot x y^{2} x y^{2} \cdot y^{2} x y^{2} x y^{m}=$ $\delta^{d-1} \cdot x y^{2} x y^{2} \cdot y^{2} x y^{2} \cdot x y^{2} x y^{2} \cdot x y^{m}$. Since $c\left(\Sigma, x y^{2} x y^{2} \cdot x y^{m}\right) \neq 0$ for any $m$, we conclude by Theorem 3.1 that $c\left(\Sigma, \delta^{d} \cdot y^{m}\right) \neq 0$, hence this open book is tight.

If $d<0$, then the curve $\alpha$ which intersects $x$ once and does not intersect $y$ is sobering for the monodromy $\delta^{d} \cdot x y^{2} x y^{2} \cdot y^{m}=h^{2 d+1} \cdot y^{m}$, as $h^{2 d+1}$ is some nonzero number of left-handed half Dehn twists around the boundary.

\footnotetext{
${ }^{4}$ Honda, Kazez, and Matić have since shown that these open books have nonvanishing contact invariant and are therefore tight if and only if $d \geq 0$.
} 
If $d=0$, then $\delta^{d} \cdot x y^{2} x y^{2} \cdot y^{m}=y^{2} x y^{2} x \cdot y^{m}$. Note that $y^{2} x y^{2} x \cdot x^{-1} y^{m}=y^{2} x y^{2+m}$, and $\left(\Sigma, y^{2} x y^{2+m}\right)=\left(\Sigma, x y^{4+m}\right)$, which is Stein fillable for $m \geq-4$. So, since $c\left(\Sigma, y^{2} x y^{2} x \cdot x^{-1} y^{m}\right) \neq 0$ for $m \geq-4$, we can conclude by Theorem 3.1 that $c\left(\Sigma, y^{2} x y^{2} x \cdot y^{m}\right) \neq 0$ for $m \geq-4$. Our methods are inconclusive when $m<-4$.

Type E If $d \geq 1$, then we have $\delta^{d} \cdot x^{m} y^{-1}=\delta^{d-1} \cdot y x^{2} y x^{2} y x^{2} y x^{2} \cdot x^{m} y^{-1}=\delta^{d-1}$. $y x^{2} y x^{2} y x^{2} y x^{2+m} y^{-1}$. And the open book $\left(\Sigma, \delta^{d-1} \cdot y x^{2} y x^{2} y x^{2} y x^{2+m} y^{-1}\right)=$ $\left(\Sigma, \delta^{d-1} \cdot x^{2} y x^{2} y x^{2} y x^{2+m}\right)=\left(\Sigma, \delta^{d-1} \cdot y x^{2} y x^{2} y x^{4+m}\right)$, which is Stein fillable for $m \geq-3$.

If $d=0$, then $\delta^{d} \cdot x^{m} y^{-1}=x^{m} y^{-1}$. Since $m \in\{-1,-2,-3\}$, the $\operatorname{arc} \alpha$ which intersects the curve $x$ once and does not intersect $y$ is sobering for this monodromy $\phi$ with

$$
i_{\text {alg }}(\alpha, \phi(\alpha))+i_{\text {geom }}(\alpha, \phi(\alpha))+i_{\delta}(\alpha, \phi(\alpha))=-1 .
$$

If $d<0$, then $\delta^{d} \cdot x^{m} y^{-1}=h^{2 k} \cdot x^{m} y^{-1}$ is then overtwisted as the arc $\alpha$ above is sobering for this monodromy by Remark 4.4.

Type $\mathbf{F}$ If $d \geq 0$, then $\delta^{d} \cdot x y^{2} x y^{2} \cdot x^{m} y^{-1}=\delta^{d} \cdot y x^{2} y x^{2} \cdot x^{m} y^{-1}=\delta^{d}$. $y x^{2} y x^{2+m} y^{-1}$. And, $\left(\Sigma, \delta^{d} \cdot y x^{2} y x^{2+m} y^{-1}\right)=\left(\Sigma, \delta^{d} \cdot x^{2} y x^{2+m}\right)=\left(\Sigma, y x^{4+m}\right)$, which is Stein fillable for $m \geq-3$.

If $d<0$, then $\delta^{d} \cdot x y^{2} x y^{2} \cdot x^{m} y^{-1}=h^{2 d+1} \cdot x^{m} y^{-1}$, and the arc $\alpha$ that was used in the proof of overtwistedness for type E monodromies where $d=0$ is also sobering for this monodromy by Remark 4.4.

Remark 4.6 In light of Remark 2.7, the proof of Theorem 4.5 shows that, for periodic monodromy, tight is equivalent to Stein fillable. Compare this with the results detailed in Section 6.

Remark 4.7 The proof also shows that tightness is equivalent to the nonvanishing of the contact invariant for all genus one, one boundary component open books which are not equivalent to $\left(\Sigma, x y^{2} x y^{2} \cdot y^{k}\right)$ where $k<-4$. In particular this implies that, for tight contact structures compatible with these open books, contact -1-surgery on a Legendrian knot is also tight. This follows from Theorem 3.1 and the fact that for any Legendrian knot $K$ in a contact three-manifold $(Y, \xi)$, there exists a compatible open book for which $K$ lies in page of the open book so that the contact framing is equal to the framing induced by the page [3, Corollary 4.23].

Remark 4.8 It is also interesting to examine our results in the context of the following question, posed by Ozsváth and Szabó in [15]: For a fibered knot $K \subset Y$, and $n$ large enough, it is clear that induced open book on $Y_{-1 / n}(K)$ is Stein fillable. But what 
is the minimal value of $n$ for which the induced open book on $Y_{-1 / n}(K)$ is tight? In the genus one case, we have answered this question in Theorem 4.5 for almost all monodromies.

\section{Spin $^{c}$ structures and Hopf invariants}

In this section, we use the fact that the manifolds $-\widehat{M}\left(0 ; 2,2,-b_{1}, \ldots,-b_{n}\right)$ are $L-$ spaces (when some $b_{i} \neq 0$ ) to compute the Hopf invariants of the contact structures associated to the open books $M\left(0 ; 2,2,-b_{1}, \ldots,-b_{n}\right)$. If $\phi$ is a monodromy, let $M(\phi)$ denote the open book $(\Sigma, \phi)$, and let $\widehat{M}(\phi)$ denote the three-manifold underlying this open book. The primary theorem of this section is the following.

Theorem 5.1 The Hopf invariant of the two-plane field associated to the contact structure given by the monodromy $\phi=x y^{2} x y^{2} \cdot x^{a_{1}} y^{-b_{1}} \ldots x^{a_{n}} y^{-b_{n}}$ is $h(\phi)=$ $1 / 2+1 / 4 \cdot \sum_{i=1}^{n}\left(b_{i}-a_{i}\right)$. Here, the $a_{i}, b_{i} \geq 0$ and $a_{i} \neq 0 \neq b_{j}$ for some $i, j$.

The proof of this theorem begins with the observation below.

Theorem 5.2 Let $\phi$ be the monodromy given by $\phi=x y^{2} x y^{2} \cdot x^{a_{1}} y^{-b_{1}} \cdots x^{a_{n}} y^{-b_{n}}$, where $a_{i} \neq 0 \neq b_{j}$ for some $i, j$, and let $Y$ denote $\widehat{M}(\phi)$. Then $c(M(\phi)) \in$ $\widehat{H F}\left(-Y, s_{c}\right)$ where $s_{c}$ is a self-conjugate $\operatorname{spin}^{c}$ structure on $-Y$. That is, $s_{c}=\overline{s_{c}}$.

Proof of Theorem 5.2 The result follows from examining the knot Floer homology $\widehat{H F K}(-Y, K)$, where $K$ is the binding of the open book decomposition $M(\phi)$ of $Y$. $\widehat{H F K}(-Y, K,-1)$ is generated by a single element which represents $c(M(\phi))$, and which is nontrivial in $\widehat{H F}\left(-Y, s_{c}\right)$. There is a conjugation symmetry in knot Floer homology [18] from which we can conclude that $\widehat{H F K}(-Y, K, 1)$ is generated by a single element in the conjugate $\operatorname{spin}^{c}$ structure $\overline{s_{c}}$. If $s_{c} \neq \overline{s_{c}}$, then the knot Floer homology in the $\operatorname{spin}^{c}$ structures $s_{c}$ and $\overline{s_{c}}$ must look like the picture in Figure 5.

However, since knot Floer homology is the $E^{1}$ term in a spectral sequence that converges to $\widehat{H F}(-Y)$, and since $c(M(\phi))$ is the sole generator of $\widehat{H F}\left(-Y, s_{c}\right)$ (recall that $-\widehat{M}\left(x y^{2} x y^{2} \cdot x^{a_{1}} y^{-b_{1}} \cdots x^{a_{n}} y^{-b_{n}}\right)$ is an $L$-space), there cannot be any nontrivial elements of $\widehat{H F K}(-Y, K, 0)$ in the $\operatorname{spin}^{c}$ structure $s_{c}$. By conjugation symmetry, this implies that there cannot be any nontrivial elements of $\widehat{H F K}(-Y, K, 0)$ in the $\operatorname{spin}^{c}$ structure $\overline{s_{c}}$. That is to say $n=0$ and the knot Floer homology in these two $\operatorname{spin}^{c}$ structures must look like that depicted in Figure 6.

Yet, this last picture is not possible either, for there is also a symmetry under orientation reversal, $\widehat{H F K}_{d}(Y, K)=\widehat{H F K}^{-d}(-Y, K)$, which respects spin ${ }^{c}$ structures but 


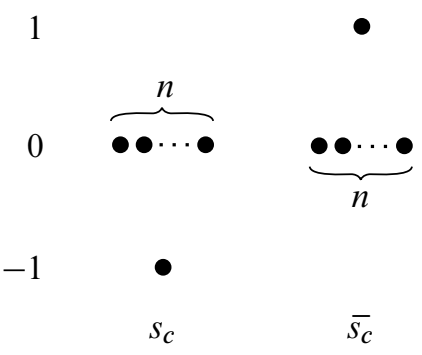

Figure 5: Possible Knot Floer homology in the $\operatorname{spin}^{c}$ structures $s_{c}$ and $\overline{s_{c}}$. The filtration is given on the left. The dots represent generators.

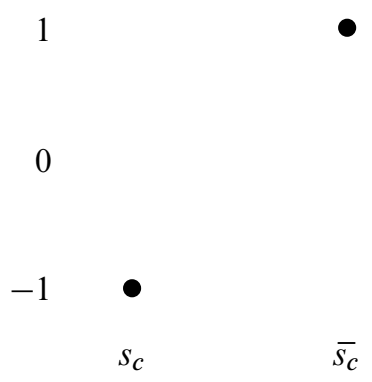

Figure 6: Another possibility for the knot Floer homology?

reverses the sign of the filtration. If our knot Floer homology $\widehat{H F K}(-Y, K)$ looks like that depicted in Figure 6, then $\widehat{H F K}(Y, K)$ looks the same, only with the filtrations changing sign. This follows from the orientation reversal symmetry and Universal Coefficient Theorem, as we are using $\mathbb{Z}_{2}$ coefficients. Then the map

$$
\widehat{H F K}(Y, K,-1) \rightarrow \widehat{H F}(Y)
$$

is nontrivial: there cannot be any higher differentials in the spectral sequence, so the generator of $\widehat{H F K}(Y, K,-1)$ must survive in $\widehat{H F}(Y)$. However, this is the statement that the contact invariant $c\left(M\left(\phi^{-1}\right)\right)$ for the corresponding contact structure on $-Y$ is nontrivial. Yet, this contact structure is overtwisted by Goodman's sobering arc criterion since

$$
\phi^{-1}=y^{b_{n}} x^{-a_{n}} \ldots y^{b_{1}} x^{-a_{1}} \cdot y^{-1} x^{-1} y^{-2} x^{-1}=y^{b_{n}} x^{-a_{n}} \ldots y^{b_{1}} x^{-a_{1}} \cdot h^{-1},
$$

so we arrive at a contradiction. 
Consequently, it must be the case that $s_{c}=\overline{s_{c}}$. This finishes the proof of Theorem 5.2. With a bit more work, it is possible to show that $\widehat{H F K}(-Y, K)$ looks like that depicted in Figure 7 in the $\operatorname{spin}^{c}$ structure $s_{c}=\overline{s_{c}}$. The $d_{1}$ differentials are shown in this figure

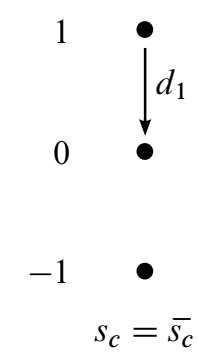

Figure 7: The knot Floer homology in the $\operatorname{spin}^{c}$ structure $s_{c}=\overline{s_{c}}$

and there can be no higher differentials in the related spectral sequence.

We now compute the absolute grading of $c\left(M\left(x y^{2} x y^{2} \cdot x^{a_{1}} y^{-b_{1}} \cdots x^{a_{n}} y^{-b_{n}}\right)\right)$. By the naturality of the contact invariant, discussed in Section 3, there is a map

$$
\begin{aligned}
F_{W}: \widehat{H F}\left(-\widehat{M}\left(x y^{2} x y^{2} \cdot x^{a_{1}} y^{-b_{1}}\right.\right. & \left.\left.\ldots x^{a_{n}} y^{-b_{n}}\right)\right) \\
& \rightarrow \widehat{H F}\left(-\widehat{M}\left(x y^{2} x y^{2} \cdot x^{a_{1}} y^{-b_{1}} \ldots x^{a_{n}} y^{-b_{n}-1}\right)\right)
\end{aligned}
$$

where $F_{W}$ (summing over all $\operatorname{spin}^{c}$ structures) maps

$$
c\left(M\left(x y^{2} x y^{2} \cdot x^{a_{1}} y^{-b_{1}} \ldots x^{a_{n}} y^{-b_{n}}\right)\right) \mapsto c\left(M\left(x y^{2} x y^{2} \cdot x^{a_{1}} y^{-b_{1}} \ldots x^{a_{n}} y^{-b_{n}-1}\right)\right) .
$$

Now, for a $\operatorname{spin}^{c}$ structure $s$ on the cobordism $W$,

$$
F_{W, \bar{s}}=\mathfrak{J} F_{W, s} \mathfrak{J}
$$

where $\mathfrak{J}: \widehat{H F}(X, t) \rightarrow \widehat{H F}(X, \bar{t})$ is the isomorphism on homology exhibited in Section 3 of [20]. Therefore,

$$
\begin{aligned}
F_{W, \bar{s}}\left(c\left(M\left(x y^{2} x y^{2} \cdot x^{a_{1}} y^{-b_{1}} \cdots x^{a_{n}} y^{-b_{n}}\right)\right)\right) & \\
& =F_{W, s}\left(c\left(M\left(x y^{2} x y^{2} \cdot x^{a_{1}} y^{-b_{1}} \cdots x^{a_{n}} y^{-b_{n}}\right)\right)\right),
\end{aligned}
$$

as the $\operatorname{spin}^{c}$ structure associated to $c\left(M\left(x y^{2} x y^{2} \cdot x^{a_{1}} y^{-b_{1}} \cdots x^{a_{n}} y^{-b_{n}}\right)\right)$ is selfconjugate. So, if $s \neq \bar{s}$, the contributions of these two maps cancel when we sum over $\operatorname{spin}^{c}$ structures. In fact the only contributions which are not cancelled out are those 
coming from the maps $F_{W, s}$, where $s=\bar{s}$ on $W$. For such $s, c_{1}(s)=-c_{1}(s)$, hence $c_{1}(s)^{2}=0$. The grading shift of the map $F_{W, s}$ is given by

$$
\frac{c_{1}(s)^{2}-3 \sigma-2 \chi}{4}
$$

which in this case is $1 / 4$ : the cobordism is gotten by two-handle addition, so $\chi=1$; in addition, this cobordism is negative definite (it cannot be positive definite as the map on $\widehat{H F}$ is nonzero, and it cannot be indefinite because all three terms in the associated surgery exact triangle involving these two manifolds are rational homology three-spheres), so $\sigma=-1$. Therefore, since $c\left(x y^{2} x y^{2} \cdot x^{a_{1}} y^{-b_{1}} \cdots x^{a_{n}} y^{-b_{n}}\right) \neq 0$, we may conclude that

$$
\begin{aligned}
\operatorname{gr}\left(c\left(M\left(x y^{2} x y^{2} \cdot x^{a_{1}} y^{-b_{1}} \ldots x^{a_{n}} y^{-b_{n}-1}\right)\right)\right) & \\
& -\operatorname{gr}\left(c\left(M\left(x y^{2} x y^{2} \cdot x^{a_{1}} y^{-b_{1}} \ldots x^{a_{n}} y^{-b_{n}}\right)\right)\right)=1 / 4 .
\end{aligned}
$$

When $n=1, a_{1}=1=b_{1},-\widehat{M}\left(x y^{2} x y^{2} \cdot x y^{-1}\right)=-L(5,1)$, and there is only one self-conjugate $\operatorname{spin}^{c}$ structure $s_{0}$ on $-L(5,1)$. Moreover, the absolute grading of the generator of $\widehat{H F}\left(-L(5,1), s_{0}\right)$ is $-1[14]$. Hence, $\operatorname{gr}\left(c\left(M\left(x y^{2} x y^{2} \cdot x y^{-1}\right)\right)\right)=-1$, and, by induction, we obtain the formula

$$
\operatorname{gr}\left(c\left(M\left(x y^{2} x y^{2} \cdot x^{a_{1}} y^{-b_{1}} \cdots x^{a_{n}} y^{-b_{n}}\right)\right)\right)=-1+1 / 4 \cdot \sum_{i=1}^{n}\left(b_{i}-a_{i}\right) .^{5}
$$

By [15], the absolute grading of the contact invariant $\operatorname{gr}(c)$ and the Hopf invariant of the corresponding two-plane field $h(\phi)$ are related by the expression $h(\phi)=-\operatorname{gr}(c)-1 / 2$. This concludes the proof of Theorem 5.1.

\section{$6 \operatorname{Tight}(\Sigma, \partial \Sigma)$ versus $\operatorname{Dehn}^{+}(\Sigma, \partial \Sigma)$}

Let $\operatorname{Tight}(\Sigma, \partial \Sigma)$ denote the set of monodromies on $\Sigma$ which correspond to tight contact structures, and let $\operatorname{Dehn}^{+}(\Sigma, \partial \Sigma)$ denote the set of monodromies whose conjugate (by some element in $\operatorname{Aut}(\Sigma, \partial \Sigma)$ ) can be expressed as the product of right-handed Dehn twists. As was mentioned before, a contact structure is Stein fillable if and only if it is compatible with some open book whose monodromy is expressible as a product of right-handed Dehn twists. We should point out that there do exist Stein fillable

\footnotetext{
${ }^{5}$ In this induction, we also need to use the maps $F_{W^{\prime}}: \widehat{H F}\left(-\widehat{M}\left(x y^{2} x y^{2} \cdot x^{a_{1}} y^{-b_{1}} \ldots x^{a_{n}} y^{-b_{n}}\right)\right) \rightarrow \widehat{H F}\left(-\widehat{M}\left(x y^{2} x y^{2} \cdot x^{a_{1}} y^{-b_{1}} \ldots x^{a_{n}-1} y^{-b_{n}}\right)\right)$, which behave in the same way as the maps $F_{W}$ described above.
} 
contact structures which are compatible with genus one, one boundary component open books, but whose monodromy cannot be taken to be in $\operatorname{Dehn}^{+}(\Sigma, \partial \Sigma)$. One such example is the contact structure compatible with the empty monodromy on $\Sigma$. After one stabilization, we see that this contact structure is Stein fillable. On the other hand, it follows from the lemma below that the empty monodromy cannot be written as a product of right-handed Dehn twists around curves in $\Sigma$. Despite this discrepancy between Stein fillable and $\operatorname{Dehn}^{+}(\Sigma, \partial \Sigma)$, an analysis of Tight $(\Sigma, \partial \Sigma)-\operatorname{Dehn}^{+}(\Sigma, \partial \Sigma)$ seems to be an appropriate first step in the identification of tight but not Stein fillable contact structures which are compatible with genus one, one boundary component open books.

This program was initiated by Honda, Kazez, and Matić in [10]. In their paper, the authors do not explicitly study $\operatorname{Tight}(\Sigma, \partial \Sigma)$. Instead, they investigate $\operatorname{Veer}(\Sigma, \partial \Sigma)$, which is the monoid of right-veering diffeomorphisms of $\Sigma$. They have since shown that $\operatorname{Veer}(\Sigma, \partial \Sigma)=\operatorname{Tight}(\Sigma, \partial \Sigma)$ when $\Sigma$ is the once-punctured torus [8; 9; 10]. The authors are able to identify infinitely many pseudo-Anosov monodromies in $\operatorname{Veer}(\Sigma, \partial \Sigma)-\operatorname{Dehn}^{+}(\Sigma, \partial \Sigma)$. Their analysis makes use of the fact that a genus one, one boundary component open book is the double cover of $S^{3}$ branched along a closed three-braid. Then, via a combination of the Rademacher function and the rotation number, they find three-braids which correspond to monodromies in $\operatorname{Veer}(\Sigma, \partial \Sigma)-\operatorname{Dehn}^{+}(\Sigma, \partial \Sigma)$.

Our result very closely parallels that of [10] but it is simpler in its statement and proof. We need only one lemma to identify infinitely many monodromies in $\operatorname{Tight}(\Sigma, \partial \Sigma)-$ $\operatorname{Dehn}^{+}(\Sigma, \partial \Sigma)$.

Lemma 6.1 If a monodromy $\phi=x^{a_{1}} y^{b_{1}} \ldots x^{a_{n}} y^{b_{n}}$ is in $\operatorname{Dehn}^{+}(\Sigma, \partial \Sigma)$ and can be written as the product of $k$ right-handed Dehn twists about homologically nontrivial simple closed curves in $\Sigma$, then

$$
\sum_{i=1}^{n} a_{i}+b_{i}=k .
$$

Writing $\delta=(x y)^{6}$, we can easily identify infinitely many $\phi \in \operatorname{Tight}(\Sigma, \partial \Sigma)-$ $\operatorname{Dehn}^{+}(\Sigma, \partial \Sigma)$ by combining Lemma 6.1 with Theorem 4.5. For instance, monodromies of the form $\phi=\delta^{k} \cdot x y^{2} x y^{2} \cdot x^{a_{1}} y^{-b_{1}} \cdots x^{a_{n}} y^{-b_{n}}$ are in $\operatorname{Tight}(\Sigma, \partial \Sigma)$ when the $a_{i}, b_{i} \geq 0, k \geq 0$, and $a_{i} \neq 0 \neq b_{j}$ for some $i, j$, by Theorem 4.5. On the other hand, according to Lemma $6.1, \phi \notin \operatorname{Dehn}^{+}(\Sigma, \partial \Sigma)$ if

$$
6+12 k+\sum_{i=1}^{n} a_{i} \leq \sum_{i=1}^{n} b_{i} .
$$

Algebraic 83 Geometric Topology, Volume 7 (2007) 
Proof of Lemma 6.1 The lemma follows from the well-known fact that $f D_{\gamma} f^{-1}=$ $D_{f(\gamma)}$ (see, for example, [13]) where, in this notation, $D_{\gamma}$ represents a right-handed Dehn twist around the curve $\gamma$ and $f: \Sigma \rightarrow \Sigma$ is an orientation-preserving homeomorphism. Keeping with this notation for right-handed Dehn twists (until the end of this section), we need only to check that, when $\alpha$ is a homologically nontrivial curve, $D_{\alpha}=D_{x}^{a_{1}} D_{y}^{b_{1}} \cdots D_{x}^{a_{n}} D_{y}^{b_{n}}$ for some collection of $a_{i}, b_{j}$ such that $\sum_{i=1}^{n} a_{i}+b_{i}=1$. This is all we need to check because the relations in $\operatorname{Aut}(\Sigma, \partial \Sigma)$ between words in $D_{x}$ and $D_{y}$ are generated by the relation $D_{x} D_{y} D_{x}=D_{y} D_{x} D_{y}$, which preserves sums of exponents.

In order to check this, observe that since $\alpha$ is homologically nontrivial, we can find an orientation-preserving homeomorphism $f$ so that $f(x)$ is isotopic to $\alpha$. Then, we have that $f^{-1} D_{x} f=D_{\alpha}$. Writing $f$ as a word in the Dehn twists $D_{x}$ and $D_{y}$, we are done.

\section{$7 \quad L$-spaces and genus one fibered knots}

Between Remark 3.9, Remark 3.10, and Lemma 3.5, we have identified three types of $L-$ spaces with genus one, one boundary component open book decompositions. These are $\widehat{M}\left(0 ; 2,2,-b_{1}, \ldots,-b_{n}\right),-\widehat{M}\left(0 ; 2,2,-b_{1}, \ldots,-b_{n}\right)$, and $\widehat{M}\left(0 ;-b_{1}, \ldots,-b_{n}\right)$. By comparing surgery diagrams, it can be shown that $-\widehat{M}\left(0 ; 2,2,-b_{1}, \ldots,-b_{n}\right)$ is homeomorphic to the three-manifold $\widehat{M}\left(-1 ; 2,2,-a_{1}, \ldots,-a_{m}\right)$ for some set of $a_{j} \in \mathbb{Z} \geq 0$ and some $a_{i} \neq 0$. We summarize these statements and more in the following theorem.

Theorem 7.1 For $b_{j} \in \mathbb{Z}^{\geq 0}$ and some $b_{i} \neq 0$, the following are $L$-spaces. Conversely, if $Y$ is an $L$-space with a genus one, one boundary component open book decomposition with pseudo-Anosov monodromy, then $Y$ takes one of the following forms:

(1) $\widehat{M}\left(0 ;-b_{1}, \ldots,-b_{n}\right)$

(2) $\widehat{M}\left(0 ; 2,2,-b_{1}, \ldots,-b_{n}\right)$

(3) $\widehat{M}\left(-1 ; 2,2,-b_{1}, \ldots,-b_{n}\right)$

Proof of Theorem 7.1 These manifolds are all $L$-spaces by arguments identical to those in the proof of Lemma 3.5. For the converse, let $M_{\phi}$ denote the mapping torus of $\phi: \Sigma \rightarrow \Sigma$. Then $M_{\phi}$ is a three-manifold with torus boundary. Let $\widehat{M}_{\phi}(p / q)$ denote the $p / q$ Dehn filling of $M_{\phi}$ with respect to some framing. Roberts [22] shows that if $\phi$ is pseudo-Anosov, then for 
- $\operatorname{trace}(\phi)>2$ and all but one Dehn filling, $\widehat{M}_{\phi}(p / q)$ has a co-orientable taut foliation, and for

- $\operatorname{trace}(\phi)<-2$, and $p / q \in(-\infty, 1)$ with respect to the framing in [22], $\widehat{M}_{\phi}(p / q)$ has a co-orientable taut foliation.

On the other hand, Ozsváth and Szabó show that $L$-spaces have no co-orientable taut foliations [17].

By Theorem 1.1, the three-manifolds with genus one, one boundary component open book decompositions whose monodromy is pseudo-Anosov with trace $>2$ are of the form $\widehat{M}\left(k ;-b_{1}, \ldots,-b_{n}\right)$, and those with trace $<-2$ are of the form $\widehat{M}\left(k ; 2,2,-b_{1}, \ldots,-b_{n}\right)$. As $k$ varies, these manifolds correspond to different Dehn fillings of mapping tori of the sort mentioned above. For trace $>2$, the $k=0$ filling is an $L$-space, and therefore has no co-orientable taut foliation. Then according to Roberts, $\widehat{M}\left(k ;-b_{1}, \ldots,-b_{n}\right)$ has a co-orientable taut foliation for $k \neq 0$, and is therefore not an $L$-space.

Suppose that $\phi$ is the monodromy of the open book $M\left(0 ; 2,2,-b_{1}, \ldots,-b_{n}\right)$. In the framing in [22], the longitude is the oriented boundary of a page of the open book and a meridian is chosen which intersects this longitude once. So, a priori, we know that $\widehat{M}\left(-1 ; 2,2,-b_{1}, \ldots,-b_{n}\right)=\widehat{M}_{\phi}(1 / m)$ for some $m$, with respect to this framing. Since $\widehat{M}\left(-1 ; 2,2,-b_{1}, \ldots,-b_{n}\right)$ is an $L-$ space, it must be true that $\widehat{M}\left(-1 ; 2,2,-b_{1}, \ldots,-b_{n}\right)$ is equal to either $\widehat{M}_{\phi}(1 / 1)$ or $\widehat{M}_{\phi}(1 / 0)$ with respect to this framing, and $\widehat{M}\left(0 ; 2,2,-b_{1}, \ldots,-b_{n}\right)$ is equal to the other. Then, the manifold $\widehat{M}\left(-k ; 2,2,-b_{1}, \ldots,-b_{n}\right)$ is equal to $\widehat{M}_{\phi}(1 / k)$ or $\widehat{M}_{\phi}(1 / 1-k)$. In either case, Roberts' results tell us that $\widehat{M}\left(-k ; 2,2,-b_{1}, \ldots,-b_{n}\right)$ must have a co-orientable taut foliation for $k \neq 1$ or 0 , and is therefore not an $L$-space.

These $L$-spaces can be used to manufacture an infinite family of hyperbolic threemanifolds with no co-orientable taut foliations. The first such examples were found by Roberts, Shareshian, and Stein [24] (see also the work of Calegari and Dunfield [2]). Consider the open book $M\left(0 ; 2,2,-b_{1}, \ldots,-b_{n}\right)$. Since $\widehat{M}\left(0 ; 2,2,-b_{1}, \ldots,-b_{n}\right)$ is an $L$-space and +1 -surgery on the binding of $M\left(0 ; 2,2,-b_{1}, \ldots, b_{n}\right)$ is an $L$-space (as it is equal to $\left.\widehat{M}\left(-1 ; 2,2,-b_{1}, \ldots,-b_{n}\right)\right)$, we can prove, using the surgery exact triangle and inductive arguments as before, the theorem below.

Theorem 7.2 $p / q$-surgery on the binding of $M\left(0 ; 2,2,-b_{1}, \ldots, b_{n}\right)$ is an $L$-space for $p / q \geq 1$. 
According to Thurston [25], if $\phi$ is pseudo-Anosov, then $M_{\phi}$ is hyperbolic. In addition, Thurston's Hyperbolic Dehn Surgery Theorem [26] guarantees that all but finitely many Dehn fillings of $M_{\phi}$ are hyperbolic as well. Let $\phi$ be the monodromy of the open book $M\left(0 ; 2,2,-b_{1}, \ldots, b_{n}\right)$. Since $\phi$ is pseudo-Anosov, and different surgeries on the binding of $M\left(0 ; 2,2,-b_{1}, \ldots, b_{n}\right)$ correspond to different Dehn fillings of $M_{\phi}$, all but finitely many of the $L$-spaces in Theorem 7.2 are hyperbolic. This family is much larger than the family of examples found in [24]. The examples in [24] can be expressed as $p / q$-surgery on the binding of manifolds of the form $M(0 ; 2,2,0,0, \ldots, 0,-1)$ for $p / q \geq 1$ and $p$ odd. These manifolds are obtained as surgeries on the components of the Borromean rings. More precisely, $p / q$-surgery on the binding of

$$
M(0 ; 2,2, \overbrace{0,0, \ldots, 0}^{m},-1)
$$

is the manifold $B(p / q, 1, m+5)$. As such, the first homology of the manifolds in [24] is generated by at most two elements. On the other hand, infinitely many of the manifolds in Theorem 7.2 have first homology generated by three elements. For instance, $p / q$-surgery on the binding of

$$
M(0 ; 2,2, \overbrace{0,0, \ldots, 0}^{m},-2)
$$

is the manifold $B(p / q, 2, m+3)$. To be fair, in [24] the authors prove that these manifolds have no taut foliations whatsoever, co-orientable or otherwise.

In [11], Kronheimer, Mrowka, Ozsváth, and Szabó exhibit an infinite family of monopole $L$-spaces (defined similarly in terms of monopole Floer homology) which are given by rational surgeries on the components of the Borromean rings for which the surgery coefficient on each component is at least 1 . A priori, it is not evident that our construction supplies any new $L$-spaces which cannot be expressed as surgeries on the Borromean rings, although it seems very likely that this is the case.

Remark 7.3 Ozsváth and Szabó conjecture that if $\left|H_{1}(Y ; \mathbb{Z})\right|=1$ and $Y$ is an $L-$ space, then $Y=n(\Sigma(2,3,5)) \# m(-\Sigma(2,3,5))$ for some integers $n$ and $m$. Examining the first homologies of the open books corresponding to the monodromies of types A-F, we find that if an integer homology three-sphere $Y$ contains a genus one fibered knot, then $Y$ is the result of some $1 / n$-surgery on one of the trefoils or on the figure eight. (For a break down of $\left|H_{1}(Y ; \mathbb{Z})\right|$ by monodromy, see [1].) If we additionally assume that $Y$ is an $L$-space then it cannot be obtained via surgery on the figure eight knot [16]. Moreover, it follows from explicit calculations in [14] that under these constraints, $Y$ can only be $S^{3}$, +1 -surgery on the right-handed trefoil, or -1-surgery 
on the left-handed trefoil. The latter two surgeries produce the manifolds $\Sigma(2,3,5)$ and $-\Sigma(2,3,5)$, respectively, yielding the following corollary:

Corollary 7.4 If $Y$ is an $L$-space, $\left|H_{1}(Y ; \mathbb{Z})\right|=1$, and $Y$ contains a genus one fibered knot, then either $Y=\Sigma(2,3,5)$ or $Y=-\Sigma(2,3,5)$.

\section{Appendix}

Here, we illustrate the proof of Lemma 3.6. The proof is not especially revealing, but we include it for the sake of completeness.

Proof of Lemma 3.6 Figure 8 gives a surgery diagram for $-\widehat{M}\left(0 ; 2,2,-b_{1}, \ldots,-b_{n}\right)$.

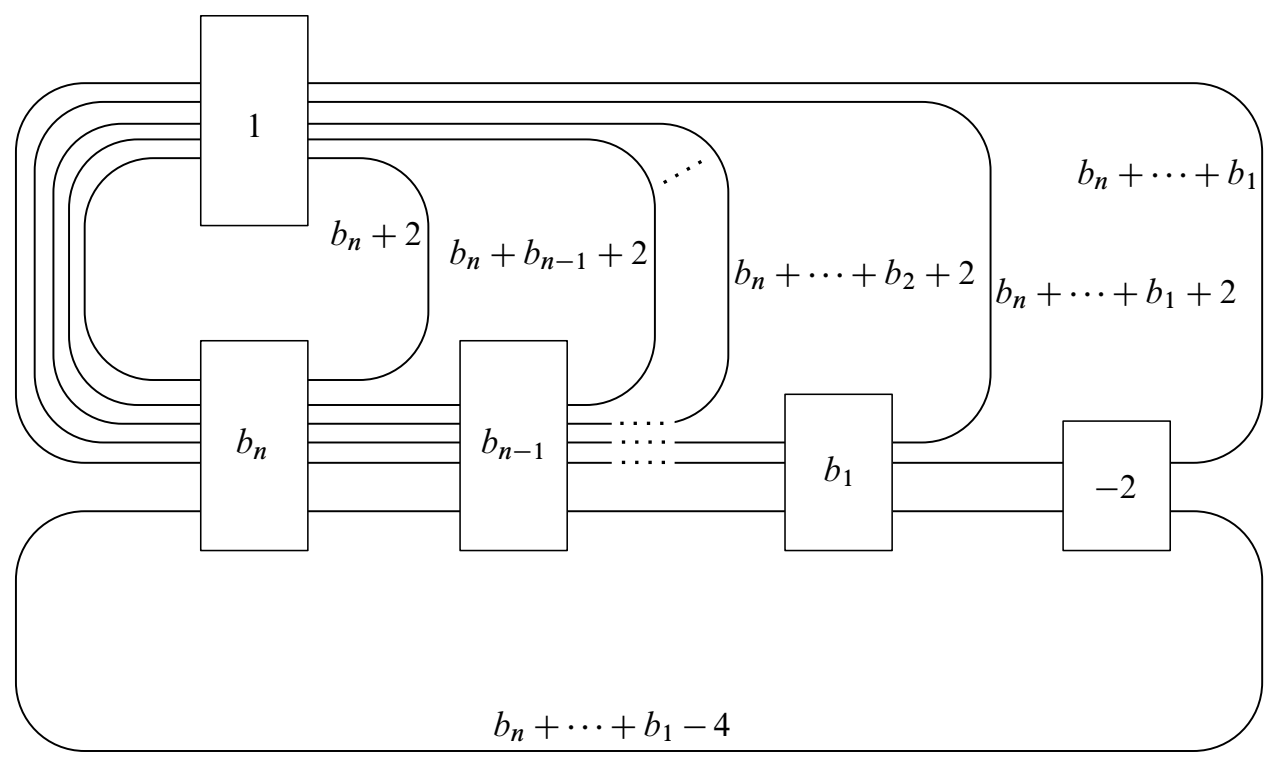

Figure 8: Surgery diagram for the $3-$ manifold $-\widehat{M}\left(0 ; 2,2,-b_{1}, \ldots,-b_{n}\right)$

The linking matrix for $-\widehat{M}\left(0 ; 2,2,-b_{1}, \ldots,-b_{n}\right)$ is given by:

$$
\left(\begin{array}{ccccc}
b_{n}+\cdots+b_{1}-4 & b_{n} & \cdots & b_{n}+\cdots+b_{1} & b_{n}+\cdots+b_{1}-2 \\
b_{n} & b_{n}+2 & \cdots & b_{n}+1 & b_{n}+1 \\
b_{n}+b_{n-1} & b_{n}+1 & \cdots & b_{n}+b_{n-1}+1 & b_{n}+b_{n-1}+1 \\
\vdots & \vdots & & \vdots & \vdots \\
b_{n}+\cdots+b_{1} & b_{n}+1 & \cdots & b_{n}+\cdots+b_{1}+2 & b_{n}+\cdots+b_{1}+1 \\
b_{n}+\cdots+b_{1}-2 & b_{n}+1 & \cdots & b_{n}+\cdots+b_{1}+1 & b_{n}+\cdots+b_{1}
\end{array}\right)
$$

Algebraic ${ }^{3} \mathcal{G}$ Geometric Topology, Volume 7 (2007) 
Then the respective linking matrices for $-\widehat{M}\left(-1 ; 2,2,-b_{1}, \ldots,-b_{n}\right)$ and $Q\left(b_{1}+\right.$ $\left.1, b_{2}, \ldots, b_{n-2}, b_{n-1}+1\right)$ are:

$$
\begin{gathered}
\left(\begin{array}{cccccc}
b_{n}+\cdots+b_{1}-4 & b_{n} & \cdots & b_{n}+\cdots+b_{1} & b_{n}+\cdots+b_{1}-2 & 1 \\
b_{n} & b_{n}+2 & \cdots & b_{n}+1 & b_{n}+1 & 1 \\
b_{n}+b_{n-1} & b_{n}+1 & \cdots & b_{n}+b_{n-1}+1 & b_{n}+b_{n-1}+1 & 1 \\
\vdots & \vdots & & \vdots & \vdots & \\
b_{n}+\cdots+b_{1} & b_{n}+1 & \cdots & b_{n}+\cdots+b_{1}+2 & b_{n}+\cdots+b_{1}+1 & 1 \\
b_{n}+\cdots+b_{1}-2 & b_{n}+1 & \cdots & b_{n}+\cdots+b_{1}+1 & b_{n}+\cdots+b_{1} & 1 \\
1 & 1 & \cdots & 1 & 1 & -1
\end{array}\right) \\
\left(\begin{array}{cccccc}
b_{n}+\cdots+b_{1}-4 & b_{n} & \cdots & b_{n}+\cdots+b_{1} & b_{n}+\cdots+b_{1}-2 & 1 \\
b_{n} & b_{n}+2 & \cdots & b_{n}+1 & b_{n}+1 & 1 \\
b_{n}+b_{n-1} & b_{n}+1 & \cdots & b_{n}+b_{n-1}+1 & b_{n}+b_{n-1}+1 & 1 \\
\vdots & \vdots & & \vdots & \vdots & \\
b_{n}+\cdots+b_{1} & b_{n}+1 & \cdots & b_{n}+\cdots+b_{1}+2 & b_{n}+\cdots+b_{1}+1 & 1 \\
b_{n}+\cdots+b_{1}-2 & b_{n}+1 & \cdots & b_{n}+\cdots+b_{1}+1 & b_{n}+\cdots+b_{1} & 1 \\
1 & 1 & \cdots & 1 & 1 & 0
\end{array}\right)
\end{gathered}
$$

Denote the determinants of these matrices by $A\left(b_{1}, \ldots, b_{n}\right), A_{-1}\left(b_{1}, \ldots, b_{n}\right)$, and $A_{0}\left(b_{1}, \ldots, b_{n}\right)$. It is clear that

$$
A_{-1}\left(b_{1}, \ldots, b_{n}\right)+A\left(b_{1}, \ldots, b_{n}\right)=A_{0}\left(b_{1}, \ldots, b_{n}\right) .
$$

By adding the last row to the previous rows, we can also see that

$$
A_{-1}\left(b_{1}, \ldots, b_{n}\right)=-A\left(b_{1}, \ldots, b_{n}+1\right) .
$$

Thus, we can write

$$
-A\left(b_{1}, \ldots, b_{n}+1\right)=A_{0}\left(b_{1}, \ldots, b_{n}\right)-A\left(b_{1}, \ldots, b_{n}\right) .
$$

If we can show that $-A\left(b_{1}, \ldots, b_{n}\right)$ is positive and increasing in the parameter $b_{n}$, then we can conclude that $A_{0}\left(b_{1}, \ldots, b_{n}\right)>0$, and the previous equation becomes

$$
\left|-A\left(b_{1}, \ldots, b_{n}+1\right)\right|=\left|A_{0}\left(b_{1}, \ldots, b_{n}\right)\right|+\left|A\left(b_{1}, \ldots, b_{n}\right)\right| .
$$

This is equivalent to

$$
\left|A_{-1}\left(b_{1}, \ldots, b_{n}\right)\right|=\left|A_{0}\left(b_{1}, \ldots, b_{n}\right)\right|+\left|A\left(b_{1}, \ldots, b_{n}\right)\right|
$$

which is the statement of Lemma 3.6. 
Lemma 8.1 We can write $A\left(b_{1}, \ldots, b_{n}\right)=-C\left(b_{1}, \ldots, b_{n}\right)$, where $C\left(b_{-} 1, \ldots, b \_n\right)$ is:

$$
\left|\begin{array}{ccccc}
b_{n}+2 & b_{n}+1 & \cdots & b_{n}+1 & b_{n}+2 \\
b_{n}+1 & b_{n}+b_{n-1}+2 & \cdots & b_{n}+b_{n-1}+1 & b_{n}+b_{n-1}+2 \\
b_{n}+1 & b_{n}+b_{n-1}+1 & \cdots & b_{n}+b_{n-1}+b_{n-2}+1 & b_{n}+b_{n-1}+b_{n-2}+2 \\
\vdots & \vdots & & \vdots & \vdots \\
b_{n}+1 & b_{n}+b_{n-1}+1 & \cdots & b_{n}+\cdots+b_{2}+2 & b_{n}+\cdots+b_{2}+2 \\
b_{n}+2 & b_{n}+b_{n-1}+2 & \cdots & b_{n}+\cdots+b_{2}+2 & b_{n}+\cdots+b_{1}+4
\end{array}\right|
$$

Proof of Lemma 8.1 By moving the first row of $A\left(b_{1}, \ldots, b_{n}\right)$ to the last row and then the first column to the last column, we have $A\left(b_{1}, \ldots, b_{n}\right)$ equals:

$$
\left|\begin{array}{ccccc}
b_{n}+2 & b_{n}+1 & \cdots & b_{n}+1 & b_{n} \\
b_{n}+1 & b_{n}+b_{n-1}+2 & \cdots & b_{n}+b_{n-1}+1 & b_{n}+b_{n-1} \\
\vdots & \vdots & & \vdots & \vdots \\
b_{n}+1 & b_{n}+b_{n-1}+1 & \cdots & b_{n}+\cdots+b_{1}+1 & b_{n}+\cdots+b_{1} \\
b_{n}+1 & b_{n}+b_{n-1}+1 & \cdots & b_{n}+\cdots+b_{1} & b_{n}+\cdots+b_{1}-2 \\
b_{n} & b_{n}+b_{n-1} & \cdots & b_{n}+\cdots+b_{1}-2 & b_{n}+\cdots+b_{1}-4
\end{array}\right|
$$

Next, we perform the following sequence of row and column operations:

(1) Subtract the third from last column from the second from last column.

(2) Subtract the third from last row from the second from last row.

(3) Subtract the third from last column from the last column.

(4) Subtract the third from last row from the last row.

After performing these operations, we see that $A\left(b_{1}, \ldots, b_{n}\right)$ becomes:

$$
\left|\begin{array}{cccccc}
b_{n}+2 & b_{n}+1 & \cdots & b_{n}+1 & 0 & -1 \\
b_{n}+1 & b_{n}+b_{n-1}+2 & \cdots & b_{n}+b_{n-1}+1 & 0 & -1 \\
\vdots & \vdots & \vdots & & \vdots & \vdots \\
b_{n}+1 & b_{n}+b_{n-1}+1 & \cdots & b_{n}+\cdots+b_{1}+2 & -1 & -2 \\
0 & 0 & \cdots & -1 & 0 & -1 \\
-1 & -1 & \cdots & -2 & -1 & -2
\end{array}\right|
$$


Next, we subtract the last column from the columns preceding it, and then subtract the last row from the rows preceding it. We obtain $A\left(b_{1}, \ldots, b_{n}\right)$ equals:

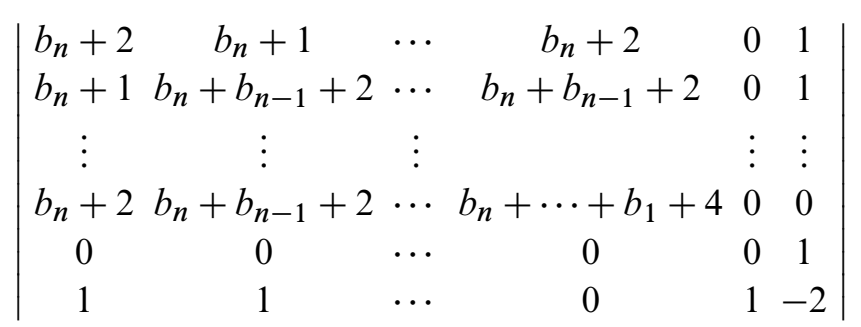

Expanding minors, we see that this is $-C\left(b_{1}, \ldots, b_{n}\right)$.

Now, we verify that $C\left(b_{1}, \ldots, b_{n}\right)$ is positive and increasing in the parameter $b_{n}$, where the $b_{j} \in \mathbb{Z}^{\geq 0}$ and some $b_{i} \neq 0$. Once we establish this, Lemma 3.6 follows immediately, as discussed above. We start with two lemmas.

Lemma 8.2 For $b_{1}, \ldots, b_{n} \geq 0, D\left(b_{1}, \ldots, b_{n}\right)>0$, where $D\left(b_{1}, \ldots, b_{n}\right)$ equals:

$$
\left|\begin{array}{ccccc}
b_{n}+1 & b_{n} & \cdots & b_{n} & b_{n} \\
b_{n} & b_{n}+b_{n-1}+1 & \cdots & b_{n}+b_{n-1} & b_{n}+b_{n-1} \\
b_{n} & b_{n}+b_{n-1} & \cdots & b_{n}+b_{n-1}+b_{n-2} & b_{n}+b_{n-1}+b_{n-2} \\
\vdots & \vdots & & \vdots & \vdots \\
b_{n} & b_{n}+b_{n-1} & \cdots & b_{n}+\cdots+b_{2}+1 & b_{n}+\cdots+b_{2} \\
b_{n} & b_{n}+b_{n-1} & \cdots & b_{n}+\cdots+b_{2} & b_{n}+\cdots+b_{1}+1
\end{array}\right|
$$

Proof of Lemma 8.2 This is clear in the case $n=1$. We proceed by induction on $n$. $D\left(b_{1}, \ldots, b_{n}\right)$ equals:

$$
\begin{aligned}
& \left|\begin{array}{cccc}
b_{n}+b_{n-1}+1 & \cdots & b_{n}+b_{n-1} & b_{n}+b_{n-1} \\
b_{n}+b_{n-1} & \cdots & b_{n}+b_{n-1}+b_{n-2} & b_{n}+b_{n-1}+b_{n-2} \\
\vdots & & \vdots & \vdots \\
b_{n}+b_{n-1} & \cdots & b_{n}+\cdots+b_{2}+1 & b_{n}+\cdots+b_{2} \\
b_{n}+b_{n-1} & \cdots & b_{n}+\cdots+b_{2} & b_{n}+\cdots+b_{1}+1
\end{array}\right| \\
& +\left|\begin{array}{ccccc}
b_{n} & b_{n} & \cdots & b_{n} & b_{n} \\
b_{n} & b_{n}+b_{n-1} & \cdots & b_{n}+b_{n-1} & b_{n}+b_{n-1} \\
b_{n} & b_{n}+b_{n-1}+b_{n-2}+1 & \cdots & b_{n}+b_{n-1}+b_{n-2} & b_{n}+b_{n-1}+b_{n-2} \\
\vdots & \vdots & & \vdots & \vdots \\
b_{n} & b_{n}+b_{n-1}+b_{n-2} & \cdots & b_{n}+\cdots+b_{2}+1 & b_{n}+\cdots+b_{2} \\
b_{n} & b_{n}+b_{n-1}+b_{n-2} & \cdots & b_{n}+\cdots+b_{2} & b_{n}+\cdots+b_{1}+1
\end{array}\right|
\end{aligned}
$$

Algebraic 83 Geometric Topology, Volume 7 (2007) 
The first summand is $D\left(b_{1}, . ., b_{n-2}, b_{n}+b_{n-1}\right)$ and is therefore $>0$ by induction. After row and column operations, we can write the second summand as:

$$
\left|\begin{array}{cccccc}
b_{n} & 0 & 0 & \cdots & 0 & 0 \\
0 & b_{n-1}+1 & b_{n-1} & \cdots & b_{n-1} & b_{n-1} \\
0 & b_{n-1} & b_{n-1}+b_{n-2}+1 & \cdots & b_{n-1}+b_{n-2} & b_{n-1}+b_{n-2} \\
\vdots & \vdots & \vdots & & \vdots & \vdots \\
0 & b_{n-1} & b_{n-1}+b_{n-2} & \cdots & b_{n-1}+\cdots+b_{2}+1 & b_{n-1}+\cdots+b_{2} \\
0 & b_{n-1} & b_{n-1}+b_{n-2} & \cdots & b_{n-1}+\cdots+b_{2} & b_{n-1}+\cdots+b_{1}+1
\end{array}\right|
$$

And this is nonnegative by induction. We have shown that we can write

$$
D\left(b_{1}, \ldots, b_{n}\right)=D\left(b_{1}, . ., b_{n-2}, b_{n}+b_{n-1}\right)+b_{n} \cdot D\left(b_{1}, \ldots, b_{n-1}\right) .
$$

We use this fact in the next lemma.

Lemma 8.3 For $b_{1}, \ldots, b_{n} \geq 0, D\left(b_{1}, \ldots, b_{n}\right)-D\left(b_{2}, \ldots, b_{n}\right) \geq 0$.

Proof of Lemma 8.3 For $n=2$, this is clear. Again, we proceed by induction. We can write

$$
\begin{aligned}
D\left(b_{1}, \ldots, b_{n}\right) & -D\left(b_{2}, \ldots, b_{n}\right) \\
& =D\left(b_{1}, . ., b_{n-2}, b_{n}+b_{n-1}\right)-D\left(b_{2}, . ., b_{n-2}, b_{n}+b_{n-1}\right) \\
& +b_{n} \cdot\left(D\left(b_{1}, \ldots, b_{n-1}\right)-D\left(b_{2}, \ldots, b_{n-1}\right)\right) .
\end{aligned}
$$

By induction, both $D\left(b_{1}, . ., b_{n-2}, b_{n}+b_{n-1}\right)-D\left(b_{2}, . ., b_{n-2}, b_{n}+b_{n-1}\right)$ and $b_{n}$. $\left(D\left(b_{1}, \ldots, b_{n-1}\right)-D\left(b_{2}, \ldots, b_{n-1}\right)\right)$ are $\geq 0$.

Now we are ready to show that, for $b_{1}, \ldots, b_{n} \geq 0, C\left(b_{1}, \ldots, b_{n}\right)$ is positive and increasing in the parameter $b_{n}$. This is clear for $n=1$. From here, we induct. $C\left(b_{1}, \ldots, b_{n}\right)$ is:

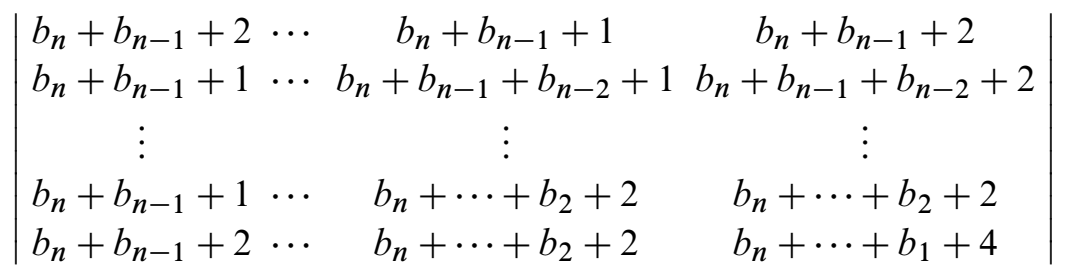

Algebraic 83 Geometric Topology, Volume 7 (2007) 


$$
+\left|\begin{array}{ccccc}
b_{n}+1 & b_{n}+1 & \cdots & b_{n}+1 & b_{n}+2 \\
b_{n}+1 & b_{n}+b_{n-1}+2 & \cdots & b_{n}+b_{n-1}+1 & b_{n}+b_{n-1}+2 \\
b_{n}+1 & b_{n}+b_{n-1}+1 & \cdots & b_{n}+b_{n-1}+b_{n-2}+1 & b_{n}+b_{n-1}+b_{n-2}+2 \\
\vdots & \vdots & & \vdots & \vdots \\
b_{n}+1 & b_{n}+b_{n-1}+1 & \cdots & b_{n}+\cdots+b_{2}+2 & b_{n}+\cdots+b_{2}+2 \\
b_{n}+2 & b_{n}+b_{n-1}+2 & \cdots & b_{n}+\cdots+b_{2}+2 & b_{n}+\cdots+b_{1}+4
\end{array}\right|
$$

The first summand is $C\left(b_{1}, \ldots, b_{n-2}, b_{n-1}+b_{n}\right)$ and is therefore $>0$ and increasing in the variable $b_{n}$ by induction. The second summand can be rewritten as:

$$
\left|\begin{array}{cccccc}
b_{n}+1 & 0 & 0 & \cdots & 0 & 1 \\
0 & b_{n-1}+1 & b_{n-1} & \cdots & b_{n-1} & b_{n-1} \\
0 & b_{n-1} & b_{n-1}+b_{n-2}+1 & \cdots & b_{n-1}+b_{n-2} & b_{n-1}+b_{n-2} \\
\vdots & \vdots & \vdots & & \vdots & \vdots \\
0 & b_{n-1} & b_{n-1}+b_{n-2} & \cdots & b_{n}+\cdots+b_{2}+1 & b_{n}+\cdots+b_{2} \\
1 & b_{n-1} & b_{n-1}+b_{n-2} & \cdots & b_{n}+\cdots+b_{2} & b_{n}+\cdots+b_{1}+1
\end{array}\right|
$$

And this is equal to $\left(b_{n}+1\right) * D\left(b_{1}, \ldots, b_{n-1}\right)-D\left(b_{2}, \ldots, b_{n-1}\right)=b_{n} \cdot D\left(b_{1}, \ldots, b_{n-1}\right)+$ $\left(D\left(b_{1}, \ldots, b_{n-1}\right)-D\left(b_{2}, \ldots, b_{n-1}\right)\right)$. By Lemma 8.2 and Lemma 8.3, this is nonnegative and increasing in $b_{n}$. This completes the proof of Lemma 3.6.

\section{References}

[1] JA Baldwin, A note on genus one fibered knots in lens spaces arXiv: math.GT/0607370

[2] D Calegari, N M Dunfield, Laminations and groups of homeomorphisms of the circle, Invent. Math. 152 (2003) 149-204 MR1965363

[3] J B Etnyre, Lectures on open book decompositions and contact structures, from: "Floer homology, gauge theory, and low-dimensional topology", Clay Math. Proc. 5, Amer. Math. Soc., Providence, RI (2006) 103-141 MR2249250

[4] J B Etnyre, K Honda, Tight contact structures with no symplectic fillings, Invent. Math. 148 (2002) 609-626 MR1908061

[5] P Ghiggini, Tight contact structures on Seifert manifolds over $T^{2}$ with one singular fibre, Algebr. Geom. Topol. 5 (2005) 785-833 MR2153106

[6] E Giroux, Convexité en topologie de contact, Comment. Math. Helv. 66 (1991) 637-677 MR1129802

[7] N Goodman, Overtwisted open books from sobering arcs, Algebr. Geom. Topol. 5 (2005) 1173-1195 MR2171807 
[8] K Honda, W Kazez, G Matić, On the contact class in Heegaard Floer homology arXiv:math.GT/0609734

[9] K Honda, W Kazez, G Matić, Right-veering diffeomorphisms of a compact surface with boundary I arXiv:math.GT/0510639

[10] K Honda, W Kazez, G Matić, Right-veering diffeomorphisms of a compact surface with boundary II arXiv:math.GT/0603626

[11] P Kronheimer, T Mrowka, P Ozsváth, Z Szabó, Monopoles and lens space surgeries arXiv:math.GT/0310164

[12] P Lisca, A Stipsicz, Contact Ozsváth-Szabó Invariants and Giroux Torsion arXiv: math. SG/0604268

[13] B Ozbagci, A I Stipsicz, Surgery on contact 3-manifolds and Stein surfaces, Bolyai Society Mathematical Studies 13, Springer, Berlin (2004) MR2114165

[14] P Ozsváth, Z Szabó, Absolutely graded Floer homologies and intersection forms for four-manifolds with boundary arXiv:math.SG/0110170

[15] P Ozsváth, Z Szabó, Heegaard Floer Homologies and Contact Structures arXiv: math.SG/0210127

[16] P Ozsváth, Z Szabó, On knot Floer homology and lens space surgeries arXiv: math.GT/0303017

[17] P Ozsváth, Z Szabó, Holomorphic disks and genus bounds, Geom. Topol. 8 (2004) 311-334 MR2023281

[18] P Ozsváth, Z Szabó, Holomorphic disks and knot invariants, Adv. Math. 186 (2004) 58-116 MR2065507

[19] P Ozsváth, Z Szabó, On the Heegaard Floer homology of branched double-covers, Adv. Math. 194 (2005) 1-33 MR2141852

[20] P Ozsváth, Z Szabó, Holomorphic triangles and invariants for smooth four-manifolds, Adv. Math. 202 (2006) 326-400 MR2222356

[21] P Ozsváth, Z Szabó, A I Stipsicz, Planar open books and Floer homology arXiv: math.SG/0504403

[22] R Roberts, Taut foliations in punctured surface bundles. I, Proc. London Math. Soc. (3) 82 (2001) 747-768 MR1816696

[23] R Roberts, Taut foliations in punctured surface bundles. II, Proc. London Math. Soc. (3) 83 (2001) 443-471 MR1839461

[24] R Roberts, J Shareshian, M Stein, Infinitely many hyperbolic 3-manifolds which contain no Reebless foliation, J. Amer. Math. Soc. 16 (2003) 639-679 MR1969207

[25] W P Thurston, The Geometry and Topology of Three-Manifolds, Princeton Univ. Math. Dept. Notes (1979) 
[26] W P Thurston, On the geometry and dynamics of diffeomorphisms of surfaces, Bull. Amer. Math. Soc. (N.S.) 19 (1988) 417-431 MR956596

[27] W P Thurston, HE Winkelnkemper, On the existence of contact forms, Proc. Amer. Math. Soc. 52 (1975) 345-347 MR0375366

Department of Mathematics, Columbia University

New York, NY 10027

baldwin@math.columbia.edu

http://www.math. columbia.edu/ baldwin

Received: 4 July $2006 \quad$ Revised: 21 March 2007 